\title{
The Szekeres Multidimensional Continued Fraction
}

\author{
By T. W. Cusick
}

\begin{abstract}
In his paper "Multidimensional continued fractions" (Ann. Univ. Sci. Budapest. Eötvös Sect. Math., v. 13, 1970, pp. 113-140), G. Szekeres introduced a new higher dimensional analogue of the ordinary continued fraction expansion of a single real number. The Szekeres algorithm associates with each $k$-tuple $\left(\alpha_{1}, \ldots, \alpha_{k}\right)$ of real numbers (satisfying $0<\alpha_{i}<1$ ) a sequence $b_{1}, b_{2}, \ldots$ of positive integers; this sequence is called a continued $k$-fraction, and for $k=1$ it is just the sequence of partial quotients of the ordinary continued fraction for $\alpha_{1}$. A simple recursive procedure applied to $b_{1}$, $b_{2}, \ldots$ produces a sequence $a(n)=\left(A_{n}^{(1)} / B_{n}, \ldots, A_{n}^{(k)} / B_{n}\right)\left(n=1,2, \ldots ; A_{n}^{(i)} \geqslant 0\right.$ and $B_{n}>U$ are integers) of simultaneous rational approximations to $\left(\alpha_{1}, \ldots, \alpha_{k}\right)$ and a sequence $c(n)=\left(c_{n 0}, c_{n 1}, \ldots, c_{n k}\right)(n=1,2, \ldots)$ of integer $(k+1)$-tuples such that the linear combination $c_{n 0}+c_{n 1} \alpha_{1}+\cdots+c_{n k} \alpha_{k}$ approximates zero. Szekeres conjectured, on the basis of extensive computations, that the sequence $a(1), a(2), \ldots$ contains all of the "best" simultaneous rational approximations to $\left(\alpha_{1}, \ldots, \alpha_{k}\right)$ and that the sequence $c(1), c(2), \ldots$ contains all of the "best" approximations to zero by the linear form $x_{0}+x_{1} \alpha_{1}+\cdots+x_{n} \alpha_{n}$. For the special case $k=2$ and $\alpha_{1}=$ $\theta^{2}-1, \alpha_{2}=\theta-1$ (where $\theta=2 \cos (2 \pi / 7)$ is the positive root of $x^{3}+x^{2}-2 x-$ $1=0$ ), Szekeres further conjectured that the 2 -fraction $b_{1}, b_{2}, \ldots$ is "almost periodic" in a precisely defined sense. In this paper the Szekeres conjectures concerning best approximations to zero by the linear form $x_{0}+x_{1}\left(\theta^{2}-1\right)+x_{2}(\theta-1)$ and concerning almost periodicity for the 2 -fraction of $\left(\theta^{2}-1, \theta-1\right)$ are proved. The method used can be applied to other pairs of cubic irrationals $\alpha_{1}, \alpha_{2}$.
\end{abstract}

1. Introduction. The ordinary continued fraction expansion of a real irrational number $\alpha$ gives a very satisfactory solution to the problem of finding closest rational approximations to $\alpha$. In particular, if we suppose $0<\alpha<1$, so that we can write the continued fraction for $\alpha$ as

$$
\alpha=\left[a_{1}, a_{2}, \ldots\right]
$$

where the $a_{i}$ 's are positive integers; and if we say $P / Q$ is a "best" rational approximation to $\alpha$ when $|Q \alpha-P|<|q \alpha-p|$ for all rationals $p / q$ with $0<q<Q$, then the sequence of convergents $p_{n} / q_{n}=\left[a_{1}, \ldots, a_{n}\right]$ for the expansion (1.1) is precisely the sequence of best approximations to $\alpha$. Furthermore, if we say $P / Q$ is a "good" rational approximation to $\alpha$ when $|Q \alpha-P|<1 / Q$, then the sequence $\left(r p_{n+1}+p_{n}\right) /\left(r q_{n+1}+q_{n}\right)$ $\left(1 \leqslant r<a_{n+2} ; n=0,1,2, \ldots\right)$ of intermediate convergents contains the sequence of good approximations to $\alpha$. (Proofs of these well-known facts can be found, for instance, in Lang $[6$, Chapter I].)

Received December 3, 1975.

AMS (MOS) subject classifications (1970). Primary 10F20, 10F10, 10F25; Secondary $10 \mathrm{~B} 45$.

Key words and phrases. Szekeres multidimensional continued fraction, ternary linear forms, Diophantine inequality, totally real cubic field. 
These ideas have two natural extensions to higher dimensions, where we consider $k>1$ different irrational numbers $\alpha_{1}, \ldots, \alpha_{k}$. First, we have the notion of simultaneous rational approximations $P_{1} / Q, \ldots, P_{k} / Q$ to $\alpha_{1}, \ldots, \alpha_{k}$. In this situation we say that we have a "best" approximation if

$$
\max _{1 \leqslant i \leqslant k}\left|Q \alpha_{i}-P_{i}\right|<\max _{1 \leqslant i \leqslant k}\left|q \alpha_{i}-p_{i}\right|
$$

for all rational $k$-tuples $p_{1} / q, \ldots, p_{k} / q$ with $0<q<Q$, and we say that we have a "good" approximation if

$$
\max _{1 \leqslant i \leqslant k} Q^{1 / k}\left|Q \alpha-P_{i}\right|<1
$$

Second, we have the notion of approximations to zero by the linear form $c_{0}+c_{1} \alpha_{1}+$ $\cdots+c_{k} \alpha_{k}$, where $c_{0}, c_{1}, \ldots, c_{k}$ are integers. In this situation we say that we have a "best" approximation if

$$
\left|c_{0}+c_{1} \alpha_{1}+\cdots+c_{k} \alpha_{k}\right|<\left|d_{0}+d_{1} \alpha_{1}+\cdots+d_{k} \alpha_{k}\right|
$$

for all integer $(k+1)$-tuples $d_{0}, d_{1}, \ldots, d_{k}$ with $\max _{1 \leqslant i \leqslant k}\left|d_{i}\right|<\max _{1 \leqslant i \leqslant k}\left|c_{i}\right|$, and we say that we have a "good" approximation if

$$
\left|c_{0}+c_{1} \alpha_{1}+\cdots+c_{k} \alpha_{k}\right| \max _{1 \leqslant i \leqslant k}\left|c_{i}\right|^{k}<1
$$

It is natural to ask whether there exists any algorithm by which the best and good approximations in these higher dimensional situations could be computed in a reasonably simple way. Such an algorithm would be a very satisfactory "multidimensional continued fraction".

Many attempts have been made to find such a multidimensional continued fraction. References to relevant papers before 1936 are given in the book of Koksma [5, pp. 50-51], and later work is covered in the compilation of reviews by LeVeque [7, pp. 193-201]. None of these proposed algorithms is very successful. For many of them, there is not even a proof that the algorithm will supply infinitely many good approximations; this is so even if we do not require such a proof in general, but only for given nontrivial examples. There is an algorithm of Minkowski [8] which does supply infinitely many good approximations to zero for any linear form $x_{0}+x_{1} \alpha_{1}+$ $x_{2} \alpha_{2}$ in which $1, \alpha_{1}, \alpha_{2}$ form a basis for a real cubic field. However, even for these special linear forms the Minkowski algorithm is quite cumbersome to use.

This paper is concerned with a multidimensional continued fraction algorithm introduced in a 1970 paper of Szekeres [9]. The Szekeres algorithm associates with each $k$-tuple $\left(\alpha_{1}, \ldots, \alpha_{k}\right)$ of real numbers, normalized so that $0<\alpha_{i}<1$ and $\alpha_{1}>\alpha_{2}>\cdots>\alpha_{k}$, a sequence $b_{1}, b_{2}, \ldots$ of positive integers; this sequence is called a continued $k$-fraction (or, for short, $k$-fraction), and for $k=1$ it turns out that the 1 -fraction is just the sequence of partial quotients of the ordinary continued fraction for $\alpha_{1}$.

A complete description of the Szekeres algorithm is given in Section 2 of this paper. Here we only remark that a simple recursive procedure applied to the integers in the $k$-fraction $b_{1}, b_{2}, \ldots$ produces a sequence $a(n)=\left(A_{n}^{(1)} / B_{n}, \ldots, A_{n}^{(k)} / B_{n}\right)$ 
( $n=1,2, \ldots ; A_{n}^{(i)} \geqslant 0$ and $B_{n}>0$ are integers) of simultaneous rational approximations to $\left(\alpha_{1}, \ldots, \alpha_{k}\right)$ and a sequence $c(n)=\left(c_{n 0}, c_{n 1}, \ldots, c_{n k}\right)(n=1,2, \ldots)$ of integer $(k+1)$-tuples such that the linear combination $c_{n 0}+c_{n 1} \alpha_{1}+\cdots+c_{n k} \alpha_{k}$ approximates zero.

Szekeres conjectured, on the basis of extensive computation of the results obtained when his algorithm was applied to various specific examples, that the sequence $a(1)$, $a(2), \ldots$ contains all of the best simultaneous rational approximations to $\left(\alpha_{1}, \ldots, \alpha_{k}\right)$ and that the sequence $c(1), c(2), \ldots$ contains all of the best approximations to zero of the linear form $x_{0}+x_{1} \alpha_{1}+\cdots+x_{n} \alpha_{n}$. He did not prove this conjecture for any $k$ fraction with $k>1$.

Let $\theta=2 \cos (2 \pi / 7)$ denote the positive root of $x^{3}+x^{2}-2 x-1=0$. Szekeres conjectured that the digits $b_{1}, b_{2}, \ldots$ of the 2 -fraction for the pair $\left(\theta^{2}-1, \theta-1\right)$ are all equal to either 1 or 2 , and that the digits of this 2 -fraction are "almost periodic" in a precisely defined sense (which is explained in Section 2 of this paper). According to computations carried out by Sved, a student of Szekeres, these conjectures are valid for the first 100,000 digits of the 2-fraction for $\left(\theta^{2}-1, \theta-1\right)$ [9, p. 138]

In this paper the above conjectures about the 2-fraction for $\left(\theta^{2}-1, \theta-1\right)$ are proved. We also prove that the Szekeres algorithm does provide all of the best approximations to zero by the linear form $x_{0}+x_{1}\left(\theta^{2}-1\right)+x_{2}(\theta-1)$. The proofs depend on some earlier work of mine [2], [3] which applies to any basis $1, \alpha_{1}, \alpha_{2}$ of a real cubic field (in fact, this work was later extended to apply to bases of any real algebraic number field [4]). This naturally raises the question whether results analogous to those proved here (for the basis $1, \theta^{2}-1, \theta-1$ of the cubic field generated by $\theta$ ) are valid for any basis $1, \alpha_{1}, \alpha_{2}$ of a real cubic field. We do not go into this question in this paper.

2. The Szekeres Algorithm. For the convenience of the reader, I give a brief explanation of the Szekeres algorithm in this section. Proofs are not given, but instead reference is made to the original paper of Szekeres [9] when necessary. The notation is mostly taken from [9].

The continued $k$-fraction, which we denote by $\left[b_{1}, b_{2}, \ldots\right]$, is a sequence (finite or infinite) of positive integers $b_{i}$ which we associate with a $k$-tuple $\left(\alpha_{1}, \ldots, \alpha_{k}\right)$ of real numbers via a certain algorithm. For simplicity in defining the algorithm, we require that

$$
1>\alpha_{1}>\alpha_{2}>\cdots>\alpha_{k}>0
$$

and that $1, \alpha_{1}, \ldots, \alpha_{k}$ are linearly independent over the rationals.

We define integers $s_{m}$ by

$$
s_{m}=b_{1}+\cdots+b_{m} \quad(m=1,2, \ldots)
$$

and we define $\epsilon(n)$ for each integer $n \geqslant 1$ by

$$
\epsilon(n)=1 \quad \text { if } n=s_{m} \text { for some } m, \quad \epsilon(n)=0 \text { otherwise. }
$$

Thus, the sequence $b_{1}, b_{2}, \ldots$ is determined if we know the sequence $\epsilon(1), \epsilon(2), \ldots$. 
In particular, if $b_{1}, b_{2}, \ldots, b_{N}$ is a finite sequence, then $\epsilon(n)=0$ for all $n>s_{N}$. We shall define the $k$-fraction $\left[b_{1}, b_{2}, \ldots\right]$ for the given $k$-tuple $\left(\alpha_{1}, \ldots, \alpha_{k}\right)$ by giving a procedure for determining the sequence $\epsilon(1), \epsilon(2), \ldots$.

First, we define a sequence of sets $\{\mathbf{A}(n, j): 0 \leqslant j \leqslant k\}(n=0,1,2, \ldots)$ of $k+1$ integer $k$-tuples

$$
\mathrm{A}(n, j)=\left(A^{(1)}(n, j), \ldots, A^{(k)}(n, j)\right) \quad(0 \leqslant j \leqslant k)
$$

and a sequence of positive integer $(k+1)$-tuples

$$
(B(n, 0), B(n, 1), \ldots, B(n, k)) \quad(n=0,1,2, \ldots)
$$

as follows. We define

$$
A^{(i)}(0, j)= \begin{cases}0, & 0 \leqslant j<i \leqslant k \\ 1, & 0 \leqslant i \leqslant j \leqslant k\end{cases}
$$

and

$$
B(0, j)=A^{(0)}(0, j)=1 \quad(0 \leqslant j \leqslant k) .
$$

Now we suppose that for some $n \geqslant 0$, we have already defined $\epsilon(n+1), \mathbf{A}(n, j)$ and $B(n, j)$ for each $j, 0 \leqslant j \leqslant k$. We also suppose that we have been given an integer $\mu(n)$, $1 \leqslant \mu(n) \leqslant k$ (the rule by which the integer $\mu(n)$ is chosen is another part of the algorithm, and is given below). Then we define

$$
\begin{aligned}
A^{(i)}(n+1,0) & =(1-\epsilon(n+1)) A^{(i)}(n, 0)+\epsilon(n+1) A^{(i)}(n, \mu(n)), \\
A^{(i)}(n+1, \mu(n)) & =A^{(i)}(n, 0)+A^{(i)}(n, \mu(n)), \\
A^{(i)}(n+1, j) & =A^{(i)}(n, j) \quad \text { for } 1 \leqslant j \leqslant k, j \neq \mu(n), 0 \leqslant i \leqslant k, \\
B(n+1, j) & =A^{(0)}(n+1, j) \quad \text { for } 0 \leqslant j \leqslant k .
\end{aligned}
$$

Proceeding in this way, we define $\mathrm{A}(n, j)$ and $B(n, j)(0 \leqslant j \leqslant k)$ inductively for $n=0,1,2, \ldots$.

We call the $k$-tuple $a(n)$ defined by

$$
\begin{array}{r}
a(n)=\left(A^{(1)}(n, \mu(n-1)) / B(n, \mu(n-1)), \ldots, A^{(k)}(n, \mu(n-1)) / B(n, \mu(n-1))\right) \\
(n=1,2, \ldots)
\end{array}
$$

the $n$th approximation fraction for the $k$-fraction of $\left(\alpha_{1}, \ldots, \alpha_{k}\right)$; we use the abbreviated notation

$$
a(n)=\mathrm{A}(n, \mu(n-1)) / B(n, \mu(n-1))
$$

in this and other similar situations. For example, we define

$$
\mathbf{P}_{n}=\mathrm{A}\left(s_{n}, 0\right), \quad Q_{n}=B\left(s_{n}, 0\right) \quad(n=1,2, \ldots)
$$

and we say that $\mathbf{P}_{n} / Q_{n}$ is the $n$th principal approximation fraction for the $k$-fraction $\left[b_{1}, b_{2}, \ldots\right]$ of $\left(\alpha_{1}, \ldots, \alpha_{k}\right)$. We define the finite $k$-fraction $\left[b_{1}, \ldots, b_{n}\right]$ by

$$
\left[b_{1}, \ldots, b_{n}\right]=\mathbf{P}_{n} / Q_{n}=a\left(s_{n}-1\right)
$$


(the second equality follows from (2.6), (2.7), (2.8) and the fact that $\epsilon\left(s_{n}\right)=1$ (from (2.3))), so the definition of the principal approximation fractions is analogous to that of the (principal) convergents $p_{n} / q_{n}=\left[a_{1}, \ldots, a_{n}\right]$ for the ordinary continued fraction expansion (1.1).

Szekeres [9, Theorem 4, p. 127 and Theorem 8, p. 130] proved that

$$
\lim _{n \rightarrow \infty} A^{(i)}\left(s_{n}, 0\right) / B\left(s_{n}, 0\right)=\alpha_{i} \text { for each } i, 1 \leqslant i \leqslant k .
$$

This, of course, is the desired analogue of the fact that $\lim _{n \rightarrow \infty} p_{n} / q_{n}=\alpha$ for the ordinary continued fraction (1.1).

Here we interrupt our discussion of the $k$-fraction briefly in order to point out how the case $k=1$ essentially reduces to the ordinary continued fraction for $\alpha_{1}$. Since $k=1$, the integer $\mu(n)$ must always be 1 . Hence, (2.6) and (2.6a) give (putting $\left.A^{(1)}(n, j)=A(n, j)\right)$

$$
\begin{aligned}
& A(n+1,0)=(1-\epsilon(n+1)) A(n, 0)+\epsilon(n+1) A(n, 1), \\
& A(n+1,1)=A(n, 0)+A(n, 1),
\end{aligned}
$$

and (2.5) and (2.6c) give similar recursions for $B(n, j)$. Using these recursions and (2.3), we successively deduce (putting $\mathbf{P}_{n}=P_{n}$, since here $\mathbf{P}_{n}$ is a 1-tuple)

$$
\begin{aligned}
& A\left(s_{n}-1,1\right)=P_{n}, \\
& A\left(s_{n}+i, 0\right)=P_{n} \quad\left(0 \leqslant i<b_{n+1}\right), \\
& A\left(s_{n}+i, 1\right)=(i+1) P_{n}+P_{n-1} \quad\left(0 \leqslant i<b_{n+1}\right) .
\end{aligned}
$$

It follows at once that $P_{n+1}=b_{n+1} P_{n}+P_{n-1}$, and a similar argument gives the same recursion for $Q_{n}$. Hence we see that the approximation fractions $P_{n} / Q_{n}$ are simply the convergents $p_{n} / q_{n}$ of the ordinary continued fraction $\left[b_{1}, b_{2}, \ldots\right]$; in view of (2.9) and (2.10), this shows that the digits $b_{1}, b_{2}, \ldots$ of the 1 -fraction for $\alpha_{1}$ are simply the partial quotients of the ordinary continued fraction for $\alpha_{1}$. Furthermore, the approximation fractions $A\left(s_{n}+i-1\right) / B\left(s_{n}+i-1\right)\left(1 \leqslant i<b_{n+1}\right)$ are simply the intermediate convergents of the continued fraction for $\alpha_{1}$ (this follows from (2.11)).

Now we continue the description of the $k$-fraction algorithm. We define a sequence of sets $\left\{\gamma_{n j}: 0 \leqslant j \leqslant k\right\}(n=0,1,2, \ldots)$ of $k+1$ positive real numbers as follows. We define

$$
\gamma_{00}=1-\alpha_{1}, \quad \gamma_{0 j}=\alpha_{j}-\alpha_{j+1} \quad(1 \leqslant j \leqslant k-1), \quad \gamma_{0 k}=\alpha_{k}
$$

Note it follows from (2.1) that $\gamma_{0 j}>0$ for each $j$. Now we suppose that for some $n \geqslant 0$ we have already defined $\gamma_{n j}$ for each $j, 0 \leqslant j \leqslant k$, and that we know the integer $\mu(n)(1 \leqslant \mu(n) \leqslant k)$ mentioned above. Then we define

$$
\epsilon(n+1)= \begin{cases}0 & \text { if } \gamma_{n 0}>\gamma_{n, \mu(n)} \\ 1 & \text { if } \gamma_{n 0}<\gamma_{n, \mu(n)}\end{cases}
$$

and we put

$$
\gamma_{n+1,0}=(1-2 \epsilon(n+1))\left(\gamma_{n 0}-\gamma_{n, \mu(n)}\right)
$$




$$
\begin{aligned}
\gamma_{n+1, \mu(n)} & =\epsilon(n+1) \gamma_{n 0}+(1-\epsilon(n+1)) \gamma_{n, \mu(n)} \\
\gamma_{n+1, j} & =\gamma_{n j} \quad \text { for } 1 \leqslant j \leqslant k, j \neq \mu(n)
\end{aligned}
$$

Since we have assumed that $1, \alpha_{1}, \ldots, \alpha_{k}$ are linearly independent over the rationals, it follows from (2.12) to (2.14b) that for each $n \geqslant 0$ and each $j, 0 \leqslant j \leqslant k$,

$$
\gamma_{n j}=c_{n j 0}+c_{n j 1} \alpha_{1}+\cdots+c_{n j k} \alpha_{k}
$$

for some unique integers $c_{n j 0}, c_{n j 1}, \ldots, c_{n j k}$.

Szekeres $[9$, p. 128] proved that

$$
\lim _{n \rightarrow \infty} \gamma_{n j}=0 \quad \text { for } 0 \leqslant j \leqslant k
$$

(the case $j=0$ of $(2.16)$ is valid only if the $k$-fraction $\left[b_{1}, b_{2}, \ldots\right]$ is not finite, but this is true here because we have assumed $1, \alpha_{1}, \ldots, \alpha_{k}$ are linearly independent). Thus, the linear combinations of $\alpha_{1}, \ldots, \alpha_{k}$ given in (2.15) provide some solutions to the problem of finding approximations to zero by the linear form $x_{0}+x_{1} \alpha_{1}+\cdots+$ $x_{k} \alpha_{k}$.

In order to complete the description of the $k$-fraction, we need only describe how the integer $\mu(n)$ is determined. We first define a partial ordering of $k$-tuples as follows: Let $\mathbf{x}=\left(x_{1}, \ldots, x_{k}\right), \mathbf{y}=\left(y_{1}, \ldots, y_{k}\right)$ and suppose that $\left|x_{i(1)}\right| \geqslant\left|x_{i(2)}\right| \geqslant \cdots \geqslant$ $\left|x_{i(k)}\right|$ and $\left|y_{j(1)}\right| \geqslant\left|y_{j(2)}\right| \geqslant \cdots \geqslant\left|y_{j(k)}\right|$ for suitable permutations of the indices $1,2, \ldots, k$. We write $\mathrm{x} \doteq \mathrm{y}$ if $\left|x_{i(r)}\right|=\left|y_{j(r)}\right|$ for $1 \leqslant r \leqslant k$ and $\mathbf{x} \prec \mathbf{y}$ if there exists an $s, 1 \leqslant s \leqslant k$, such that $\left|x_{i(s)}\right|<\left|y_{j(s)}\right|$ and $\left|x_{i(r)}\right|=\left|y_{j(r)}\right|$ for $1 \leqslant r<s$. Next we define

$$
\mathbf{V}(n, j)=\frac{\mathbf{A}(n, j)}{B(n, j)}-\frac{\mathbf{A}(n, 0)}{B(n, 0)} \quad(1 \leqslant j \leqslant k ; n=0,1,2, \ldots)
$$

The integer $\mu(n)(1 \leqslant \mu(n) \leqslant k)$ is defined to be the largest integer $h$ such that for every $j, 1 \leqslant j \leqslant k$, we have

$$
\mathrm{V}(n, j) \prec \mathrm{V}(n, h) \quad \text { or } \quad \mathrm{V}(n, j) \doteq \mathrm{V}(n, h) .
$$

In particular, it follows from (2.4) that always

$$
\mu(0)=k \text {. }
$$

This completes the description of the $k$-fraction algorithm. To find the $k$-fraction $\left[b_{1}, b_{2}, \ldots\right]$ for $\left(\alpha_{1}, \ldots, \alpha_{k}\right)$ we begin at stage $n=0$ with $\mathbf{A}(0, j)$ and $B(0, j)$ (given by (2.4) and (2.5)), $\gamma_{0 j}$ (given by (2.12)), $\epsilon(1)$ (given by (2.13)) and $\mu(0)$ (given by (2.19)). At stage $n=m$ we have determined $\mathrm{A}(m, j)$ and $B(m, j), \gamma_{m j}, \epsilon(m+1)$ and $\mu(m)$. This enables us to determine $\mathbf{A}(m+1, j)$ and $B(m+1, j)$ from (2.6) through (2.6c), $\gamma_{m+1, j}$ from (2.14) through (2.14b), $\epsilon(m+2)$ from (2.13) and $\mu(m+1)$ from the definition, using (2.17) and (2.18) with $n=m+1$. In this way we successively determine the digits $b_{i}$ by using (2.3) and the definition (2.2) of $s_{i}$.

The above method for determining the integer $\mu(n)$ is an important feature of the Szekeres algorithm. The choice of $\mu(n)$ is motivated by geometric considerations (see 
Szekeres [9, pp. 129-130]), the idea being that the selection of $\mu(n)$ in the way indicated should ensure that the algorithm does not stray away from good solutions to the problems of simultaneous rational approximations and of linear form approximations to zero. In this respect the Szekeres algorithm is specifically designed to try to overcome the known defects of various earlier multidimensional continued fractions (see the discussion in Szekeres [9, pp. 113-117]).

We give a further discussion here of the geometrical content of the Szekeres algorithm in the case $k=2$. The facts summarized here will be important for the work of Section 4 below.

Define the straight lines $\Gamma_{n j}\left(x_{1}, x_{2}\right)=0$ in the $x_{1}-x_{2}$ plane by

$$
\Gamma_{n j}\left(x_{1}, x_{2}\right)=c_{n j 0}+c_{n j 1} x_{1}+c_{n j 2} x_{2}=0 \quad(n=0,1,2, \ldots ; 0 \leqslant j \leqslant 2),
$$

where the coefficients are given in (2.15). Define the approximation triangle $\Delta_{n}$ ( $n=$ $0,1,2, \ldots)$ to be the region in the $x_{1}-x_{2}$ plane determined by the inequalities

$$
\Gamma_{n j}\left(x_{1}, x_{2}\right) \geqslant 0, \quad 0 \leqslant j \leqslant 2
$$

It is clear that $\Delta_{n}$ contains the point with coordinates $\left(\alpha_{1}, \alpha_{2}\right)$, by $(2.15)$ and the fact that $\gamma_{n j}>0$ always. Szekeres [9, p. 126] showed that the vertices of the triangle $\Delta_{n}$ are just the points with coordinates $\mathbf{A}(n, j) / B(n, j), 0 \leqslant j \leqslant 2$. It follows from (2.6) to (2.6c) that $\Delta_{n+1}$ has two vertices in common with $\Delta_{n}$. Further $[9$, p. 126], the new vertex $\mathrm{A}(n+1, \mu(n)) / B(n+1, \mu(n))$ of $\Delta_{n+1}$ is on the side of $\Delta_{n}$ which is the line segment from $\mathbf{A}(n, 0) / B(n, 0)$ to $\mathbf{A}(n, \mu(n)) / B(n, \mu(n))$.

Two typical consecutive approximation triangles $\Delta_{n}$ and $\Delta_{n+1}$ are shown in Figure 1. Note that the line $\Gamma_{n i}\left(x_{1}, x_{2}\right)=0$ contains the vertices $\mathbf{A}(n, j) / B(n, j)$ for $0 \leqslant j \leqslant 2, j \neq i[9$, formula (3.12), p. 122], and the common vertices are $\mathbf{A}(n, 1) / B(n, 1)$ and $A(n, 2) / B(n, 2)$ (by $(2.6)$ and $(2.6 \mathrm{~b})$, since $\epsilon(n+1)=1$ and $\mu(n)=1$ in Figure 1).

The rule for determining the integer $\mu(n)$ can be explained geometrically as follows [9, pp. 129-130]: Define the span of the line segment from a point $\left(x_{1}, x_{2}\right)$ to a point $\left(y_{1}, y_{2}\right)$ to be $\max \left(\left|x_{1}-y_{1}\right|,\left|x_{2}-y_{2}\right|\right)$. The integer $\mu(n)$ is chosen so that the new vertex $\mathrm{A}(n+1, \mu(n)) / B(n+1, \mu(n))$ of $\Delta_{n+1}$ lies on whichever of the two sides of $\Delta_{n}$ containing $A(n, 0) / B(n, 0)$ has the larger span. (If the spans are equal, we compare the size of the remaining projections of the two sides on the coordinate axes, as explained in discussing (2.18) above.) The precise definition of the new vertex of $\Delta_{n+1}$ is (see $[9$, formula (5.3), p. 126] or (2.6a) and (2.6c))

$$
\frac{\mathrm{A}(n+1, \mu(n))}{B(n+1, \mu(n))}=\frac{\mathbf{A}(n, 0)+\mathbf{A}(n, \mu(n))}{B(n, 0)+B(n, \mu(n))} .
$$

This is a two-dimensional generalization of the process whereby two consecutive Farey fractions $p / q$ and $r / s$ are "added" to form a new Farey fraction $(p+r) /(q+s)$.

Szekeres pursues this analogy in $[9$, Section $5, \mathrm{pp} .126-129]$, but we require only (2.22). 


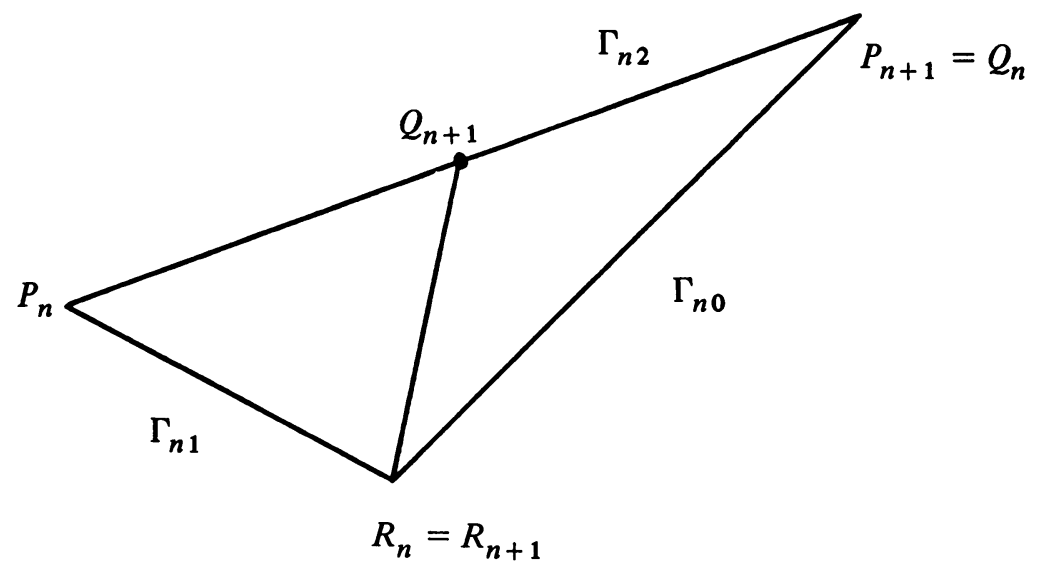

$$
\begin{aligned}
& \epsilon(n+1)=1, \mu(n)=1 \\
& \Delta_{n}=\Delta P_{n} Q_{n} R_{n}, \Delta_{n+1}=\Delta P_{n+1} Q_{n+1} R_{n+1} \\
& P_{i}=A(i, 0) / B(i, 0), Q_{i}=A(i, 1) / B(i, 1), R_{i}=A(i, 2) / B(i, 2) \text { for } i=n, n+1
\end{aligned}
$$

FIGURE 1. Typical approximation triangles $\Delta_{n}$ and $\Delta_{n+1}$

We shall also need the following lemma for the work of Section 4:

LEMmA 1. The matrix

$$
\left[\begin{array}{lll}
B(n, 0) & A^{(1)}(n, 0) & A^{(2)}(n, 0) \\
B(n, 1) & A^{(1)}(n, 1) & A^{(2)}(n, 1) \\
B(n, 2) & A^{(1)}(n, 2) & A^{(2)}(n, 2)
\end{array}\right]
$$

has determinant \pm 1 . Its inverse matrix is

$$
\left[\begin{array}{lll}
c_{n 00} & c_{n 10} & c_{n 20} \\
c_{n 01} & c_{n 11} & c_{n 21} \\
c_{n 02} & c_{n 12} & c_{n 22}
\end{array}\right]
$$

where the $c_{n j k}$ are defined by (2.15).

Proof. The lemma is contained in results of [9, pp. 121-122].

Now we turn to the particular 2-fraction for $\left(\alpha_{1}, \alpha_{2}\right)=\left(\theta^{2}-1, \theta-1\right)$, where $\theta=2 \cos (2 \pi / 7)$ is the positive root of $x^{3}+x^{2}-2 x-1=0$. In the remainder of the paper, we give a detailed discussion of this 2 -fraction. The notations

$$
\alpha=\theta^{2}-1, \quad \beta=\theta-1
$$

and

$$
\left[d_{1}, d_{2}, \ldots\right]=2 \text {-fraction for }(\alpha, \beta)
$$

will be used from now on.

The 2-fraction for $(\alpha, \beta)$ is discussed in detail by Szekeres [9, pp. 138-140] . 
He reports that his student $M$. Sved used a computer to calculate more than 100,000 of the digits $d_{1}, d_{2}, \ldots$. All of these digits were either 1 or 2 ; and it was found that, apart from some irregularities at the beginning, the sequence $d_{1}, d_{2}, \ldots$ followed an almost periodic pattern made up of repetitions of the digit blocks $a=2121$ and $b=$ 1121. To be precise, the pattern of the digits $d_{i}$ can be described as follows: Let $s_{0}$ denote the digit pattern $a=2121$ and let $s_{1}$ denote the digit pattern $b=1121$. Let $s_{j}^{n}$ denote $n$ repetitions of the block of digits $s_{j}$ and define

$$
\begin{aligned}
& s_{2}=s_{1} s_{0}=11212121, \quad s_{3}=s_{2}^{8} s_{1}, \quad s_{4}=s_{3} s_{2}, \\
& s_{5}=s_{4}^{14} s_{3}, \quad s_{6}=s_{5} s_{4}, \quad s_{7}=s_{6} s_{5}, \quad s_{8}=s_{7}^{3} s_{6}, \quad s_{9}=s_{8}^{5} s_{7}, \\
& s_{10}=s_{9} s_{8}, \quad s_{11}=s_{10} s_{9}, \quad s_{12}=s_{11} s_{10}, \quad s_{13}=s_{12} s_{11} .
\end{aligned}
$$

Then, apart from the initial irregularities, the first 100,000 digits $d_{i}$ are a suitable section of $s_{12}$ (which, by a little calculation, has 146,896 digits).

The "exponents" $\beta(i)$ in the equations $s_{i+1}=s_{i}^{\beta(i)} s_{i-1}(i=1,2, \ldots)$ of (2.23) are

$$
1,8,1,14,1,1,3,5,1,1,1,1 \text {. }
$$

Szekeres [9, pp. 138-139] points out that the digits (2.24) are just the first dozen partial quotients in the ordinary continued fraction expansion of the number $\eta$ given by

$$
\eta=\frac{3 \log \theta}{\log (\theta+2)-2 \log \theta}=.89935351 \ldots
$$

He gives a geometric argument which shows heuristically why a connection between the partial quotients of $\eta$ and the pattern of the blocks $s_{i}$ might be expected.

In Section 5 of the present paper we give a proof that the 2 -fraction digits $d_{i}$ are indeed almost periodic, as suggested by (2.23). The relevance of the number $\eta$ in connection with the pattern of the $s_{i}$ 's is also proved.

3. Good Approximations to Zero by $x+\alpha y+\beta z$. One of our goals (which we will attain in Section 4 , after the preliminaries of this section) is to show that for $k=2, \alpha_{1}=\alpha=\theta^{2}-1, \alpha_{2}=\beta=\theta-1$ (as defined in Section 2), the linear forms $\gamma_{n 0}(n=0,1,2, \ldots)$ defined by $(2.15)$ include all of the best approximations to zero by the linear form $x_{0}+x_{1} \alpha+x_{2} \beta$. Since we shall mainly be concerned with $\gamma_{n j}$ for $j=0$, we modify the notation (2.15) so that the middle subscript in $c_{n j i}$ is omitted when $j=0$. Thus, we define

$$
\gamma_{n 0}=c_{n 0}+c_{n 1} \alpha+c_{n 2} \beta
$$

We further define the coefficient 3-tuples $c(n)$ of $\gamma_{n 0}$ by

$$
c(n)=\left(c_{n 0}, c_{n 1}, c_{n 2}\right) \text {. }
$$

In my papers [2] and [4] I gave a general algorithm for finding all of the solutions in integers $x_{0}, x_{1}, \ldots, x_{n}$ not all zero of 


$$
\left|x_{0}+x_{1} \alpha_{1}+\cdots+x_{n} \alpha_{n}\right| \max _{1 \leqslant i \leqslant n}\left|x_{i}\right|^{n}<c
$$

where $1, \alpha_{1}, \ldots, \alpha_{n}$ are a basis for a real algebraic number field of degree $n$ and $c$ is any fixed positive real number. Paper [4] discusses this algorithm in general terms, but [2] and especially its sequel [3] give a detailed study of the inequality

$$
\left|x+\theta y+\theta^{2} z\right| \max \left(y^{2}, z^{2}\right)<1
$$

Of course,

$$
x+\theta y+\theta^{2} z=x+y+z+\alpha z+\beta y,
$$

so the solutions of (3.3) provide all of the good approximations to zero by the linear form $x_{0}+x_{1} \alpha+x_{2} z$.

We shall need to relate the solutions of (3.3) to the coefficients $c(n)$ of $\gamma_{n 0}$ given in (3.2). Some results from [2] and [3] which we require are given without proof in this section. Our notation mostly follows that of those papers.

Let $F$ denote the cubic field defined by $\theta$, and let $\theta^{\prime}=2 \cos (4 \pi / 7), \theta^{\prime \prime}=$ $2 \cos (6 \pi / 7)$ be the conjugates of $\theta$. Then (note that many decimals in this paper are truncated, not rounded off)

$$
\theta=1.24697960 \ldots, \quad \theta^{\prime}=-.44504186 \ldots, \quad \theta^{\prime \prime}=-1.80193773 \ldots .
$$

Since $F$ is a cyclic or Abelian field, $\theta^{\prime}$ and $\theta^{\prime \prime}$ belong to $F$. Also, $1, \theta, \theta^{2}$ is an integral basis for $F$ and $\theta, \theta^{\prime}$ is a pair of fundamental units for $F$. We let $\varphi=1 / \theta^{\prime}$ for later convenience, so $\theta, \varphi$ is also a pair of fundamental units for $F$.

Given any unit $\omega$ of norm 1 in $F, \omega^{-1}=\theta^{m} \varphi^{n}$ for some unique integers $m$ and $n$. Define

$$
R(m, n)=\omega^{-1}=\theta^{m} \varphi^{n}
$$

and define integers $b(m, n), g(m, n), k(m, n)$ by

$$
|R(m, n)|=b(m, n)+k(m, n) \alpha+g(m, n) \beta
$$

$\left(|R(m, n)|\right.$ is uniquely expressible in this form since $1, \theta, \theta^{2}$ is an integral basis for $\left.F\right)$. We note that the notations (3.4) and (3.5) are not the same as in papers [2] and [3] . In those papers (see [2, p. 169] and [3, p. 980]) $R(m, n)$ was defined as $\theta^{m+1} \varphi^{n}$, and integers $b_{m}^{(n)}, g_{m}^{(n)}, k_{m}^{(n)}$ were defined by $R(m, n)=b_{m}^{(n)}+g_{m}^{(n)} \theta+k_{m}^{(n)} \theta^{2}$ (this linear form can vary in sign, whereas the form defined in (3.5) is always positive). In particular, the following relations hold between the two notations:

$$
\begin{gathered}
b_{m-1}^{(n)}=(-1)^{n}(b(m, n)-g(m, n)-k(m, n)), \quad g_{m-1}^{(n)}=(-1)^{n} g(m, n), \\
k_{m-1}^{(n)}=(-1)^{n} k(m, n) .
\end{gathered}
$$

Of course, the definitions (3.4) and (3.5) are more convenient in this paper, and we use (3.6) to translate results from [2] and [3] into the notation used here.

Next we define

$$
S(m, n)=|R(m, n)| \max \left(k(m, n)^{2}, g(m, n)^{2}\right) .
$$


Notice that $S(m, n)$ here is the same as $S(m-1, n)$ in the notation of [2] and [3], because of (3.6). For each integer $n$, let $v(n)$ denote the value of $m$ with the property that $S(v(n), n)<S(m, n)$ for all integers $m \neq v(n)$. If, as in [2, Section 4], the values of $S(m, n)$ are tabulated in a rectangular array with the integers $m$ arranged on a vertical axis and the integers $n$ arranged on a horizontal axis, then $S(v(n), n)$ is the smallest entry in one of the columns of the array. We are only interested in good approximations, and for these $S(m, n)<1$; thus by (3.4) we are only interested in values of $m$ and $n$ such that $\left|\theta^{m} \varphi^{n}\right|<1$. It turns out [2, formula (19), p. 171] that, apart from a finite number of exceptions, $S(m, n)<1$ occurs only in the second quadrant $(m \geqslant 0$, $n \leqslant 0$ ). In fact, even more is true: apart from finitely many exceptions, every solution of

$$
\left|x_{0}+x_{1} \alpha+x_{2} \beta\right| \max \left(x_{1}^{2}, x_{2}^{2}\right)<1.3
$$

corresponds to some $S(m, n)$ with $m \geqslant 0, n<0$ [3, p. 980]. For the convenience of the reader, Table 1 of [2], which gives part of the $S(m, n)$ array for $m \geqslant 1,-1 \geqslant n$ is reproduced in this paper.

Using the work of [2] and [3], we are able to determine the locations in the $S(m, n)$ array of the good approximations, that is of the values of $S(m, n)$ which are less than 1 . We can also deduce the locations of the best approximations as a subset of the good ones. In order to do these things, we require some more notation and results from [2] and [3].

If $\alpha$ is any function of $\theta, \theta^{\prime}$ and $\theta^{\prime \prime}$, let $\alpha^{\prime}$ and $\alpha^{\prime \prime}$ denote the numbers obtained by replacing $\theta, \theta^{\prime}, \theta^{\prime \prime}$ by $\theta^{\prime}, \theta^{\prime \prime}, \theta$ and $\theta^{\prime \prime}, \theta, \theta^{\prime}$, respectively, in the expression for $\alpha$. Thus, if $\omega^{-1}=\theta^{m} \varphi^{n}$, then $\omega^{\prime-1}=\theta^{\prime m} \varphi^{\prime n}$ and $\omega^{\prime \prime-1}=\theta^{\prime \prime m} \varphi^{\prime \prime}$. Now let $n$ be any fixed integer and put $\omega_{m}^{-1}=\theta^{m} \varphi^{n}$; define $u(n)$ to be the value of $m$ with the property that

$$
|| \omega_{u(n)}^{\prime \prime} / \omega_{u(n)}^{\prime}|-1|<|| \omega_{m}^{\prime \prime} / \omega_{m}^{\prime}|-1|
$$

for all integers $m \neq u(n)$. The function $u(n)$ is easy to calculate, as the following lemma [2, Lemma 5, p. 170] shows:

Lemma 2. Define $E_{1}=\theta \theta^{\prime 2}$ and $E_{2}=\left|\varphi \varphi^{\prime 2}\right|$. The integer $u(n)$ is equal to the unique integer $m$ which satisfies

$$
\frac{\log \left(2\left(1+E_{1}\right)^{-1}\right)}{\log E_{1}}<m+\frac{n \log E_{2}}{\log E_{1}}<1+\frac{\log \left(2\left(1+E_{1}\right)^{-1}\right)}{\log E_{1}} .
$$

Let $E(n)$ denote $\omega_{u(n)}^{\prime \prime} / \omega_{u(n)}^{\prime}=\left(\theta \theta^{\prime 2}\right)^{u(n)}\left(\varphi \varphi^{\prime 2}\right)^{n}$; this function will be very important later on. Note that it follows simply from the definition of $u(n)$ that $[2$, formula (17), p. 170]

$$
2 \theta^{\prime 2}<|E(n)|<2 \theta^{-1}
$$

for every $n$. So we define

$$
E_{-}=2 \theta^{\prime 2} \approx .39612452, \quad E_{+}=2 \theta^{-1} \approx 1.60387548
$$




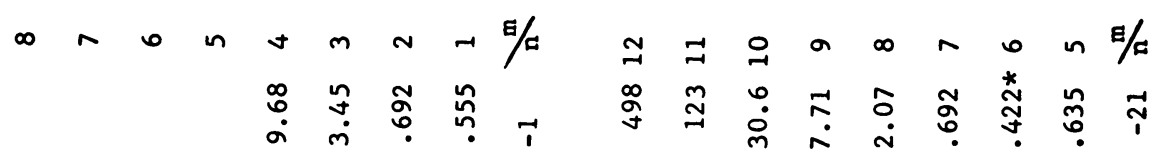

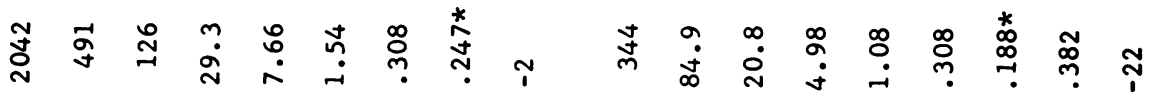

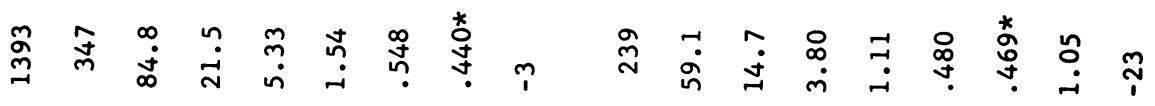
。

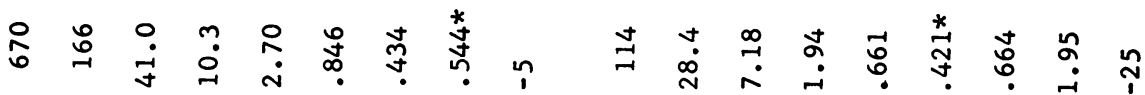

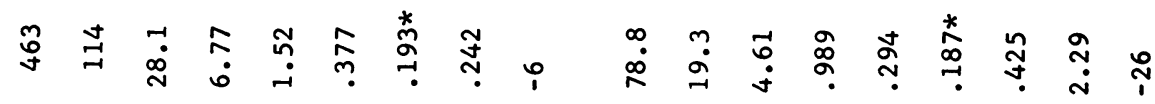

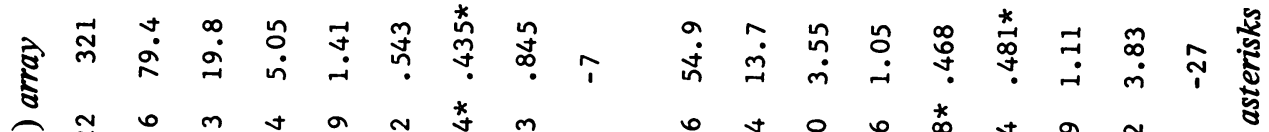
§ సิ 芯

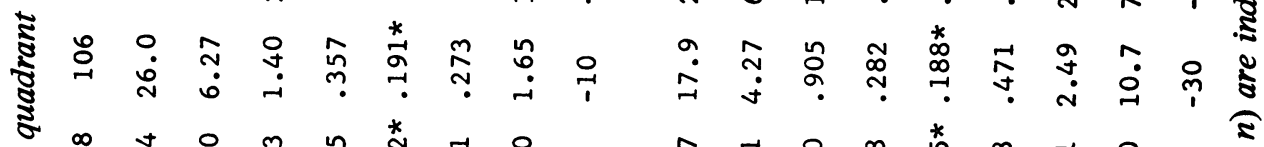
ఫ๊̋ ڤ 要节

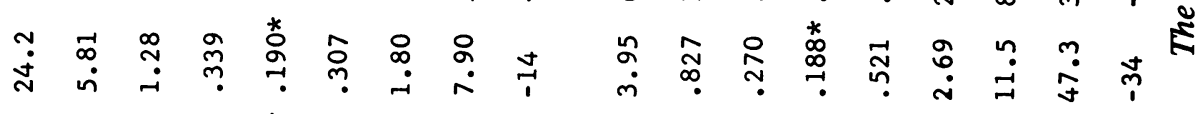

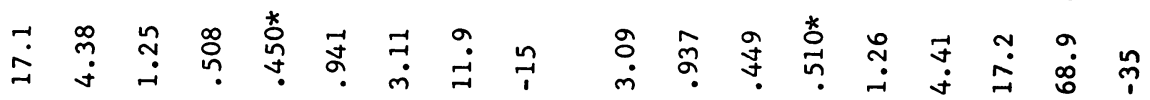
品 品

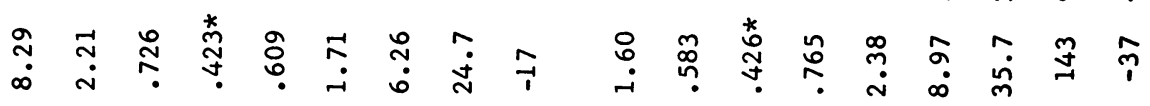

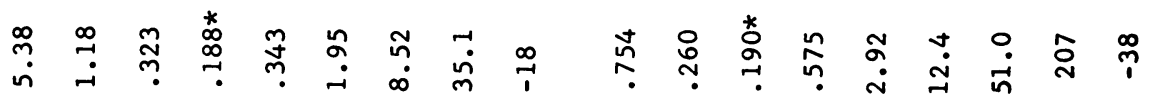

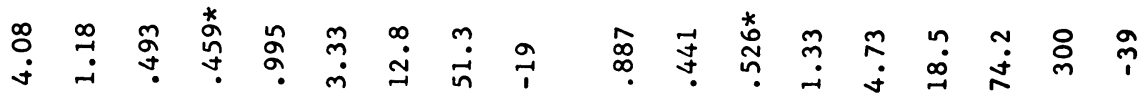

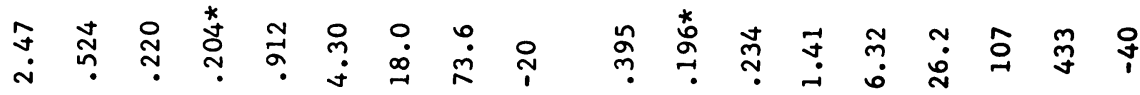


It is proved in [3, pp. 982-983] that $S(u(n)+m, n)<S(u(n)+m+1, n)$ holds for all sufficiently large $m>0$ and is false for all sufficiently large $|m|, m<0$. In fact,

$$
S(u(n)+m, n) \rightarrow \infty \text { as } m \rightarrow+\infty \text { or } m \rightarrow-\infty \text {; }
$$

this follows at once from a generalization of formulas (13) and (14) of [3, p. 985]. A more detailed but straightforward examination of the same generalized formulas gives the following:

THEOREM 1. For $n<0$ and $|n|$ sufficiently large, the inequality $S(u(n)+m, n)$ $<1$ is possible only for $m=-1,0,1$ or 2 .

Theorem 1 provides the desired localization of the good approximations $S(m, n)$. Since it follows from Lemma 2 that

$$
\lim _{n \rightarrow-\infty} u(n) / n=-\log E_{2} / \log E_{1} \approx-.263,
$$

Theorem 1 says that the good approximations all lie along a certain line in the second quadrant of the $S(m, n)$ array. The pattern is clear in Table 1 , where the numbers $S(u(n), n)$ are indicated by asterisks. Notice that because of (3.6), these numbers are one line lower than the numbers marked similarly in [2, Table 1, p. 178] or [3, Table 1, p. 981].

The localization of the best approximations is more complicated, and is described in Theorem 2 below. We say that $S(m, n)$ is a best approximation if the coefficients $b(m, n), g(m, n), k(m, n)$ (defined by (3.5)) give a best linear form approximation to zero, as defined in Section 1.

Define

$$
T_{m, n}=\max (|g(u(n)+m, n)|,|k(u(n)+m, n)|) .
$$

Notice that $T_{m, n}$ here is the same as $T_{m-1, n}$ in the notation of [3], because of (3.6). We require the following lemma about $T_{m, n}$ later on.

LEMMA 3. If $n<0$ and $|n|$ is sufficiently large, then

$$
\text { for } n \text { even, } T_{m, n}>T_{0, n} \text { for all } m \neq 0,1 \text {, }
$$

and

$$
\text { for } n \text { odd, } T_{m, n}>T_{0, n} \text { for all } m \neq 0,1,2 \text {. }
$$

Proof. Assertion (3.9) is [3, Lemma 7, Corollary 1, p. 989] and assertion (3.10) is [3, Lemma 9, Corollary 1, p. 990].

Of course, we must replace the notation $T_{m, n}$ of [3] by $T_{m+1, n}$ in order to state Lemma 3 in the notation of the present paper.

THEOREM 2. For $n<0$ and $|n|$ sufficiently large, $S(u(n)+m, n)$ can be a best approximation only in the following cases:

(i) $n$ even, $m=-1$ or 0 ,

(ii) $n$ even, $m=1$ and $E(n)>\left|\theta^{3} \theta^{\prime}\right|^{-1} \approx 1.1588$,

(iii) $n$ odd, $m=-1$ or 0 ,

(iv) $n$ odd, $m=1$ and $-E(n)>\theta^{-1} \approx .8019$,

(v) $n$ odd, $m=2$ and $-E(n)>\theta^{2} \approx 1.5550$. 
Note. We later show (in the proof of Theorem 6 in Section 4) that Theorem 2 remains true if case (v) is deleted.

Proof. Since the best approximations are a subset of the good approximations, it follows from Theorem 1 that we need only prove the inequalities on $E(n)$ in cases (ii), (iv), (v) of Theorem 2. Recall that by the definition of $E(n), E(n)>0$ if and only if $n$ is even.

For $m=1,|R(u(n), n)|=\theta^{u(n)}|\varphi|^{n}<|R(u(n)+1, n)|=\theta^{u(n)+1}|\varphi|^{n}$, so by definition $S(u(n)+1, n)$ cannot be a best approximation unless $T_{1, n}<T_{0, n}$. But by [3, Lemma 7, Corollary 2, p. 989], this inequality holds for $n$ even only if $E(n)>$ $\left|\theta^{3} \theta^{\prime}\right|^{-1}$; and by [3, Lemma 9, Corollary 2, p. 990], the inequality holds for $n$ odd only if $-E(n)>\theta^{-1}$. This does cases (ii) and (iv).

For $m=2$, reasoning as above shows that $S(u(n)+2, n)$ cannot be a best approximation unless $T_{2, n}<T_{0, n}$. But by [3, Lemma 9, Corollary 2, p. 990], this inequality holds for $n$ odd only if $-E(n)>\theta^{2}$; and by Lemma 3, (3.9), we always have $T_{2, n}>T_{0, n}$ for $n$ even. Thus case (v) is the only remaining possibility for a best approximation, and the proof of Theorem 2 is complete.

It is natural to expect that for each $n$, any best approximations $S(m, n)$ will be close to the entry $S(v(n), n)$ in the $n$th column of the $S(m, n)$ array. This is so, and a quantitative expression of this fact is contained in the next theorem [3, Theorem 1, p. 983] (we must replace $u(n)$ in [3] by $u(n)+1$, because of (3.6)).

THEOREM 3. For each even integer $n<0, v(n)$ equals either $u(n)$ or $u(n)-1$. For each odd integer $n<0, v(n)$ equals either $u(n)$ or $u(n)+1$.

Thus Theorems 2 and 3 show that, at least for $|n|$ sufficiently large, whenever $S(m, n)$ is a best approximation, the inequality $|m-v(n)| \leqslant 2$ holds.

We will need the following lemmas concerning the function $u(n)$ for the work of the next section.

Lemma 4. Suppose $n<0$; then $u(n)$ increases as $n$ decreases. There are no more than four consecutive values of $n$ for which $u(n)$ has the same value. Whenever $u(n)$ takes on a given value, it has that value for at least three consecutive values of $n$. For $n<0, u(n)$ takes on every nonnegative integer value.

Proof. This is [3, Lemma 2, p. 984].

LEMMA 5. We have

$$
\begin{array}{ll}
u(n-1)=u(n) & \text { if and only if } E_{-}<|E(n)|<2\left|\theta^{\prime \prime}\right|^{-1} \approx 1.1099, \\
u(n-1)=u(n)+1 & \text { if and only if } 2\left|\theta^{\prime \prime}\right|^{-1}<|E(n)|<E_{+} .
\end{array}
$$

In the former case, $E(n-1)=E(n) / \theta^{2} \theta^{\prime} \approx-1.4450 E(n)$; in the latter case, $E(n-1)$ $=E(n) / \theta^{2} \theta^{\prime \prime} \approx-.3569 E(n)$.

We also have

$$
\begin{array}{ll}
u(n-2)=u(n) & \text { if and only if } E_{-}<|E(n)|<2 \theta^{3} \theta^{\prime 2} \approx .7681, \\
u(n-2)=u(n)+1 & \text { if and only if } 2 \theta^{3} \theta^{\prime 2}<|E(n)|<E_{+} .
\end{array}
$$

In the former case, $E(n-2)=E(n) / \theta^{4} \theta^{2} \approx 2.0881 E(n)$; in the latter case, $E(n-2)$ $=E(n) / \theta^{3} \approx .5157 E(n)$. 
Proof. The assertions in (3.11) are given in [3, formula (39), p. 990] and the assertions in (3.12) are given in [3, formula (23), p. 986]. The statements about $E(n)$ are based on the fact that $E(n)=\theta^{u(n)+2 n} \theta^{\prime 2 u(n)+n}$, which is an immediate consequence of the definitions $E(n)=\left(\theta \theta^{\prime 2}\right)^{u(n)}\left(\varphi \varphi^{\prime 2}\right)^{n}, \varphi=1 / \theta^{\prime}$.

Next we define

$$
G=\theta^{\prime \prime}-\theta \approx-3.04891734, \quad K=\theta^{\prime \prime}-\theta^{\prime} \approx-1.35689587,
$$

so that

$$
G^{\prime} \approx 1.69202147, \quad G^{\prime \prime} \approx 1.35689587, \quad K^{\prime} \approx 3.04891734, \quad K^{\prime \prime} \approx-1.69202147 .
$$

The following lemma will be used in the calculations of Section 4.

LEMMA 6. For every integer $m$,

$$
g(m, n)=\frac{1}{7}\left(G \theta^{m} \varphi^{n}+G^{\prime} \theta^{\prime m} \varphi^{\prime n}+G^{\prime \prime} \theta^{\prime \prime m} \varphi^{\prime n}\right)
$$

and

$$
k(m, n)=\frac{1}{7}\left(K \theta^{m} \varphi^{n}+K^{\prime} \theta^{\prime m} \varphi^{\prime n}+K^{\prime \prime} \theta^{\prime \prime m} \varphi^{\prime \prime n}\right)
$$

Proof. This is just part of [2, Lemma 4, pp. 169-170]; $D$ in that lemma is 7 in the situation we have here.

4. The 2-Fraction for $(\alpha, \beta)$. It turns out (we prove it below) that in the 2fraction for $(\alpha, \beta)$ all of the positive real numbers $\gamma_{n j}(n=0,1,2, \ldots ; 0 \leqslant j \leqslant 2)$ are units of the cubic field $F$ defined by $\theta$. Thus each $\gamma_{n j}$ is of the form $(-1)^{m(2)} \theta^{m(1)} \varphi^{m(2)}$ for some integers $m(1)$ and $m(2)$ (recall $\varphi=1 / \theta^{\prime}<0$, so the factor $(-1)^{m(2)}$ ensures that $\gamma_{n j}>0$ has the correct sign). Table 2 gives the values of each $\gamma_{n j}$ and of the numbers $\epsilon(n+1), \mu(n)$ for $0 \leqslant n \leqslant 38$.

The main result which we prove in this section is that the triples $\left(\gamma_{n 0}, \gamma_{n 1}, \gamma_{n 2}\right)$ $(n=0,1,2, \ldots)$ can be divided into a finite number of classes in the following way: For each $n$ we have

$$
\begin{gathered}
\gamma_{n 0}=(-1)^{m(2)} \theta^{m(1)} \varphi^{m(2)}, \quad \gamma_{n 1}=(-1)^{m(2)+s} \theta^{m(1)+r} \varphi^{m(2)+s} \\
\gamma_{n 2}=(-1)^{m(2)+u} \theta^{m(1)+t} \varphi^{m(2)+u}
\end{gathered}
$$

for some integers $m(1), m(2), r, s, t$ and $u$. We put two triples in the same class if the integers $r, s, t, u, \epsilon(n+1)$ and $\mu(n)$ are the same for both triples. It turns out that for $n \geqslant 4$ each of the triples $\left(\gamma_{n 0}, \gamma_{n 1}, \gamma_{n 2}\right)$ belongs to one of ten classes; further, for $n \geqslant 15$ eight classes are sufficient (and necessary) to contain every triple. The ten classes are listed in Table 3. The last column in Table 2 gives the class to which the corresponding triple belongs. The sequence of class labels in Table 2 appears to become periodic for $n \geqslant 15$, We shall eventually show (in Section 5) that in fact this sequence is almost periodic.

Let us now indicate the dependence on $n$ of the integers $m(1), m(2), r, s, t, u$ in (4.1) by putting $m(1)=m(1, n), m(2)=m(2, n), r=r(n)$, etc. 
TABLE 2. Values of $\gamma_{n j}$ in the 2-fraction for $(\alpha, \beta)$

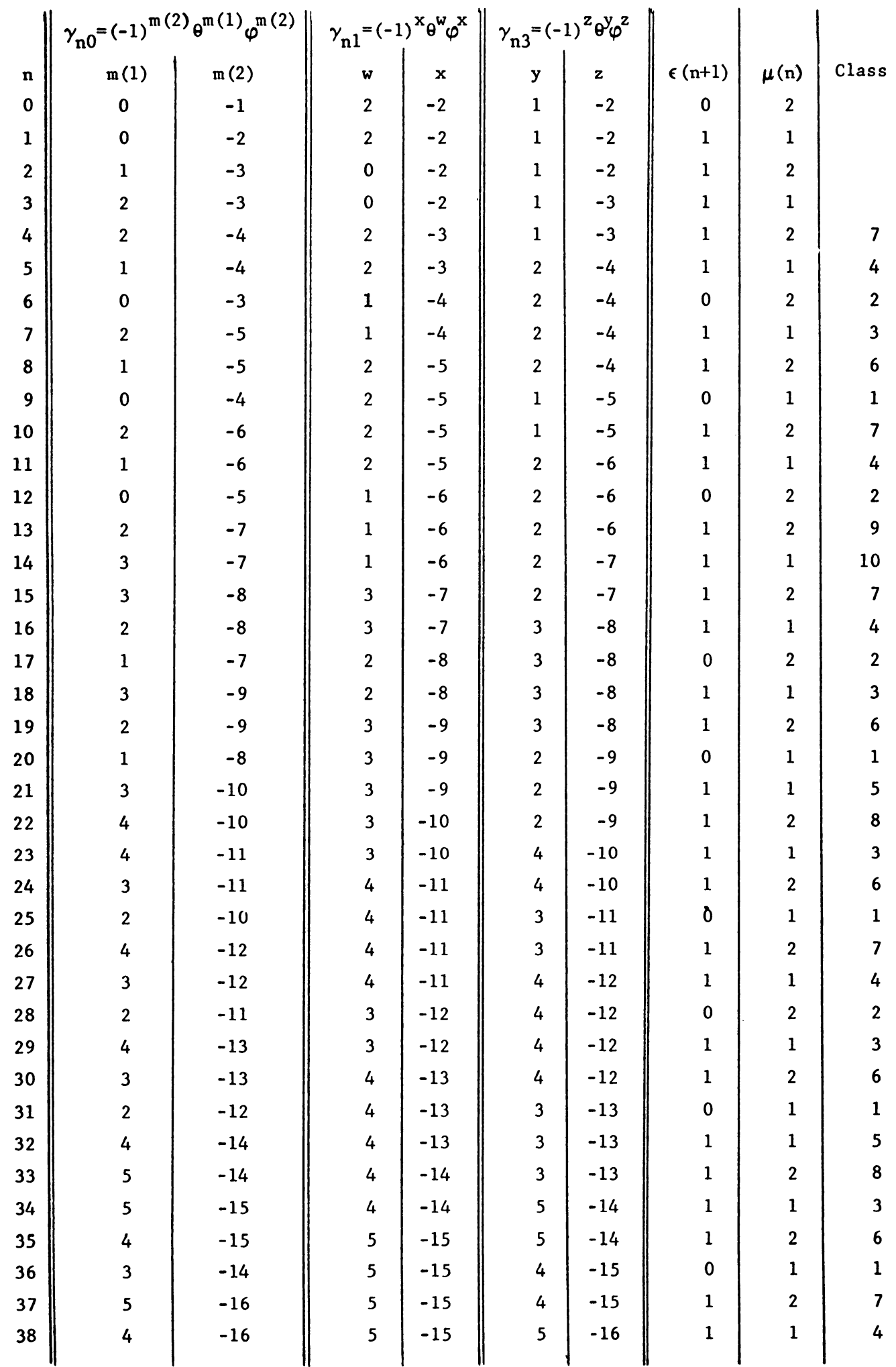


TABLE 3. Labels for classes of triples $\gamma_{n 0}=(-1)^{m(2)} \theta^{m(1)} \varphi^{m(2)}$, $\gamma_{n 1}=(-1)^{m(2)+s} \theta^{m(1)+r} \varphi^{m(2)+s}, \gamma_{n 2}=(-1)^{m(2)+u} \theta^{m(1)+t} \varphi^{m(2)+u}$ \begin{tabular}{c|r|r|r|r|r|r|} 
Class & \multicolumn{1}{|c|}{$r$} & $\mathrm{~s}$ & $\mathrm{t}$ & $\mathrm{u}$ & $\epsilon(\mathrm{n}+1)$ & $\mu(\mathrm{n})$ \\
1 & 2 & -1 & 1 & -1 & 0 & 1 \\
2 & 1 & -1 & 2 & -1 & 0 & 2 \\
3 & -1 & 1 & 0 & 1 & 1 & 1 \\
4 & 1 & 1 & 1 & 0 & 1 & 1 \\
5 & 0 & 1 & -1 & 1 & 1 & 1 \\
6 & 1 & 0 & 1 & 1 & 1 & 2 \\
7 & 0 & 1 & -1 & 1 & 1 & 2 \\
8 & -1 & 0 & -2 & 1 & 1 & 2 \\
9 & -1 & 1 & 0 & 1 & 1 & 2 \\
10 & -2 & 1 & -1 & 0 & 1 & 1
\end{tabular}

TABLE 4. Determination of the class to which the triple $\tau_{n+1}=\left(\gamma_{n+1,0}, \gamma_{n+1,1}, \gamma_{n+1,2}\right)$ belongs from the class of the triple $\tau_{n}$

\begin{tabular}{|c|c|c|c|}
\hline $\begin{array}{l}\text { Class } \\
\text { of } \tau_{n}\end{array}$ & $\begin{array}{c}\text { Allowable values } \\
\text { of } \mu(n+1)\end{array}$ & $\begin{array}{l}\text { Class } \\
\text { of } \tau_{n+1}\end{array}$ & $\left|\gamma_{n+1,0} / \gamma_{n, 0}\right|$ \\
\hline \multirow[t]{2}{*}{1} & 1 & 5 & $\theta^{2} \varphi^{-2}$ \\
\hline & 2 & 7 & $\theta^{2} \varphi^{-2}$ \\
\hline \multirow[t]{2}{*}{2} & 1 & 3 & $\theta^{2} \varphi^{-2}$ \\
\hline & 2 & 9 & \\
\hline 3 & 2 & 6 & $\theta^{-1}$ \\
\hline 4 & 2 & 2 & $\theta^{-1}|\varphi|$ \\
\hline 5 & 2 & 8 & $\theta$ \\
\hline 6 & 1 & 1 & $\theta^{-1}|\varphi|$ \\
\hline 7 & 1 & 4 & $\theta^{-1}$ \\
\hline 8 & 1 & 3 & $|\varphi|^{-1}$ \\
\hline 9 & 1 & 10 & $\theta$ \\
\hline 10 & 2 & 7 & $|\varphi|^{-1}$ \\
\hline
\end{tabular}

We also define the triple $\tau_{n}$ by

$$
\tau_{n}=\left(\gamma_{n 0}, \gamma_{n 1}, \gamma_{n 2}\right) \quad(n=0,1,2, \ldots) .
$$

It is clear from (2.14) to (2.14b) that if we are given the integers $m(1, n), m(2, n)$, $r(n), s(n), t(n), u(n), \epsilon(n+1)$ and $\mu(n)$, then the real numbers $\gamma_{n+1,0}, \gamma_{n+1,1}$ and 
$\gamma_{n+1,2}$ are determined. For the particular 2 -fraction which we are considering, an easy calculation using (2.13) shows that the integer $\epsilon(n+2)$ is also determined, except possibly when the triple $\tau_{n}$ defined by (4.2) belongs to class 5 of Table 3. Since the numbers $\gamma_{n+1, j}(0 \leqslant j \leqslant 2)$ are known and are units in the cubic field $F$, it follows that (4.1) is valid with $n+1$ in place of $n$, and so the integers $m(1, n+1), m(2, n+1)$, $r(n+1), s(n+1), t(n+1)$ and $u(n+1)$ are all determined. Only $\mu(n+1)$ (which determines $\epsilon(n+2)$ via $(2.13)$ if $\epsilon(n+2)$ is not already known) remains to be specified. A much more detailed study of the 2 -fraction for $(\alpha, \beta)$ shows (see below for the proofs) that in fact $\mu(n+1)$ has only one allowable value unless the triple $\tau_{n}$ is in class 1 or 2 , in which case both values of $\mu(n+1)$ can occur. It is also the case that the allowable values of $\mu(n+1)$ always give triples $\tau_{n+1}$ which are in one of the ten classes listed in Table 3. This accounts for the fact that only finitely many different classes occur.

Table 4 gives the allowable values of $\mu(n+1)$ for each of the ten classes, and the thereby determined classes to which the triples $\tau_{n+1}$ belong. Table 4 also gives

$$
\left|\gamma_{n+1,0} / \gamma_{n, 0}\right|=\theta^{m(1, n+1)-m(1, n)}|\varphi|^{m(2, n+1)-m(2, n)},
$$

which depends only on the class of the triple $\tau_{n}$.

The following theorem summarizes the results we have stated so far in this section.

THEOREM 4. The numbers $\gamma_{n j}(n=0,1,2, \ldots ; 0 \leqslant j \leqslant 2)$ are all cubic units. The triples $\tau_{n}=\left(\gamma_{n 0}, \gamma_{n 1}, \gamma_{n 2}\right)(n=4,5,6, \ldots)$ are each in one of the ten classes listed in Table 3. The class to which the triple $\tau_{n+1}(n \geqslant 4)$ belongs is determined either by the class of $\tau_{n}$ alone (if $\tau_{n}$ is in any of the classes except 1 and 2) or by the class of $\tau_{n}$ and the value of $\mu(n+1)$ (if $\tau_{n}$ is in class 1 or 2$)$; these determinations are listed in Table 4.

Proof. We can verify directly that the numbers $\gamma_{n j}(0 \leqslant j \leqslant 2)$ are units for $0 \leqslant n \leqslant 4$ and that the triple $\tau_{4}$ is in class 7 (see Table 2). Given these facts, the first sentence of the theorem follows from the second, and the second sentence follows from the third. Thus, to prove the theorem we need only prove the last sentence. A complete proof involves ten cases, one for each class of triples. We give details of only two of the ten cases here. The calculations for the two cases which we fully work out are completely typical, so that the work in the eight omitted cases follows the same pattern.

Calculations for Class 1 . We first assume that each number in the triple $\tau_{n}$ is a unit, so that in particular we have from (3.4) that

$$
\gamma_{n 0}=\left|R\left(u\left(n^{*}\right)+i, n^{*}\right)\right|=\theta^{u\left(n^{*}\right)+i}|\varphi|^{n *}
$$

for some integers $n^{*}$ and $i$. From now on we always use the notation $n^{*}$ to denote the integer (depending on $n$ ) such that (4.3) is valid. For example, $\gamma_{n 0}$ is in class 1 for $n=9,20,25,31,36, \ldots$ and the corresponding values for $n^{*}$ (from Table 2) are $n^{*}=-4,-8,-10,-12,-14$, respectively.

We call the integer $i$ which appears in (4.3) the index of $\gamma_{n 0}$. It will turn out that for each class, the set of different indices of the numbers $\gamma_{n 0}$ in the class is finite. 
TABLE 5. Description of subclasses by index and inequalities on $E(n)^{*}$

\begin{tabular}{|c|c|c|c|c|}
\hline $\begin{array}{c}\text { Subclass } \\
\text { of } \tau_{n}\end{array}$ & $\begin{array}{c}\text { Parity } \\
\text { of } n^{*}\end{array}$ & $\begin{array}{l}\text { Index } \\
\qquad i\end{array}$ & Restrictions on $E\left(n^{*}\right)$ & $\begin{array}{l}\text { Class } \\
\text { of } \tau_{n+1}\end{array}$ \\
\hline la & even & -1 & $\theta^{4}\left|\theta^{\prime}\right| \approx 1.0761<E\left(n^{*}\right)<1.6039 \approx E_{+}$ & 5 \\
\hline $1 b$ & even & -1 & $\theta\left|\theta^{\prime}\right| \approx .5550<\mathrm{E}\left(\mathrm{n}^{*}\right)<1.0761 \approx \theta^{4}\left|\theta^{\prime}\right|$ & 7 \\
\hline $1 c$ & even & -2 & $E_{-} \approx .3961<E\left(n^{*}\right)<.5550 \approx \theta\left|\theta^{\prime}\right|$ & 5 \\
\hline $2 a$ & odd & -1 & $\theta^{-1} \approx .8019<-\mathrm{E}\left(\mathrm{n}^{*}\right)<1.5550 \approx \theta^{2}$ & 3 \\
\hline $2 b$ & odd & -1 & $\theta^{2} \approx 1.5550<-E\left(n^{*}\right)<1.6039 \approx E_{+}$ & 9 \\
\hline $3 a$ & odd & 0 & $E_{-} \approx .3961<-E_{\left(n^{*}\right)}<.8019 \approx \theta^{-1}$ & 6 \\
\hline $3 b$ & odd & 1 & $\theta^{-1} \approx .8019<-E\left(\mathrm{n}^{*}\right)<1.6039 \approx \mathrm{E}_{+}$ & 6 \\
\hline $4 a$ & even & -1 & $E_{-} \approx .3961<E\left(n^{*}\right)<.5550 \approx \theta\left|\theta^{\prime}\right|$ & 2 \\
\hline $4 b$ & even & 0 & $\theta^{-3}\left|\theta^{\prime}\right|^{-1} \approx 1.1588<\mathrm{E}\left(\mathrm{n}^{*}\right)<1.6039 \approx \mathrm{E}_{+}$ & 2 \\
\hline 5 & even & 0 & $\theta\left|\theta^{\prime}\right| \approx .5550<\mathrm{E}\left(\mathrm{n}^{*}\right)<1.1588 \approx \theta^{-3}\left|\theta^{\prime}\right|^{-1}$ & 8 \\
\hline $6 a$ & odd & -1 & $E_{-} \approx .3961<-E\left(n^{*}\right)<.8019 \approx \theta^{-1}$ & 1 \\
\hline $6 \mathrm{~b}$ & odd & 0 & $\theta^{-1} \approx .8019<-E\left(n^{*}\right)<1.6039 \approx E_{+}$ & 1 \\
\hline $7 a$ & even & 0 & $\mathrm{E}_{-} \approx .3961<\mathrm{E}\left(\mathrm{n}^{*}\right)<.5550 \approx \theta\left|\theta^{\prime}\right|$ & 4 \\
\hline $7 b$ & even & 1 & $\theta^{-3}\left|\theta^{\prime}\right|^{-1} \approx 1.1588<\mathrm{E}\left(\mathrm{n}^{*}\right)<1.6039 \approx \mathrm{E}_{+}$ & 4 \\
\hline 8 & even & 1 & $\theta\left|\theta^{\prime}\right| \approx .5550<\mathrm{E}\left(\mathrm{n}^{*}\right)<1.1588 \approx \theta^{-3}\left|\theta^{\prime}\right|^{-1}$ & 3 \\
\hline 9 & odd & 0 & $\theta^{-1} \approx .8019<-E\left(n^{*}\right)<.8272 \approx 2 \theta^{-4}$ & 10 \\
\hline 10 & odd & 1 & $\theta^{-1} \approx .8019<-E\left(n^{*}\right)<.8272 \approx 2 \theta^{-4}$ & 7 \\
\hline
\end{tabular}

Table 5 contains a complete list of the different possible indices (the proof that the list is complete is given later on, in Theorem 5). In particular, for class 1 the only possible indices are -1 and -2 .

The parity of the integer $n^{*}$ is determined by the class to which $\tau_{n}$ belongs (see Table 5). Furthermore, for $\tau_{n}$ in a given class, the index of $\gamma_{n 0}$ has a given value if and only if $E\left(n^{*}\right)$ satisfies certain inequalities (see Table 5; here $E(n)=\left(\theta \theta^{\prime 2}\right)^{u(n)}$. $\left(\varphi \varphi^{\prime 2}\right)^{n}$ is the function defined in Section 3). It is convenient to divide each class into subclasses according to the index of $\gamma_{n 0}$ and the class of $\tau_{n+1}$; this is done in Table 5 .

Since $\varphi<0$, the definition of $E(n)$ implies that $E\left(n^{*}\right)$ is $>0$ if and only if $n^{*}$ is even. This is reflected in the inequalities for $E\left(n^{*}\right)$ in Table 5.

Since $\tau_{n}$ is in class 1 , we have $\epsilon(n+1)=0$ and $\mu(n)=1$ from Table 3. Hence, by $(2.14)$ to $(2.14 \mathrm{~b})$ and the rest of the data for class 1 in Table 3, we have

$$
\gamma_{n+1,0}=\theta^{m(1)}|\varphi|^{m(2)}-\theta^{m(1)+2}|\varphi|^{m(2)-1}, \quad \gamma_{n+1,1}=\gamma_{n 1}, \quad \gamma_{n+1,2}=\gamma_{n 2},
$$

with $m(1)$ and $m(2)$ determined by (4.3). Thus using the identity $1-\theta^{2}|\varphi|^{-1}=$ $\theta^{2} \varphi^{-2}$, we get

$$
\begin{gathered}
\gamma_{n+1,0}=\theta^{m(1)+2}|\varphi|^{m(2)-2}, \quad \gamma_{n+1,1}=\theta^{m(1)+2}|\varphi|^{m(2)-1}, \\
\gamma_{n+1,2}=\theta^{m(1)+1}|\varphi|^{m(2)-1} .
\end{gathered}
$$

It follows from (2.13) and (4.4) that $\epsilon(n+2)=1$, so the Eqs. (4.4) and Table 3 
imply that $\tau_{n+1}$ is either in class 5 or in class 7 , depending on whether $\mu(n+1)$ equals 1 or 2 , respectively. This already gives all of the statements in Theorem 4 which apply to $\tau_{n}$ in class 1 . However, we shall prove more (namely, some of the inequalities for $E\left(n^{*}\right)$ in Table 5 which apply in the various subclasses of class 1 for indices -1 and -2 ); we do this because the results are needed below, and the necessary calculations are representative of those needed to establish Theorem 4 for $\tau_{n}$ in any class other than 1 or 2 .

We first assume that $\tau_{n}$ is in subclass $1 \mathrm{a}$ or $1 \mathrm{~b}$, so $n^{*}$ is even and the index of $\gamma_{n 0}$ is -1 . This means (using (4.3) with $i=-1$, (3.5) and the notation in (3.1))

$$
\gamma_{n 0}=c_{n 0}+c_{n 1} \alpha+c_{n 2} \beta=c_{n 0}+k\left(u\left(n^{*}\right)-1, n^{*}\right) \alpha+g\left(u\left(n^{*}\right)-1, n^{*}\right) \beta .
$$

Also, by the definition of class 1 in Table 3 we have (using (4.5) and the notation in (2.15))

$$
\gamma_{n 1}=c_{n 10}+k\left(u\left(n^{*}\right)+1, n^{*}-1\right) \alpha+g\left(u\left(n^{*}\right)+1, n^{*}-1\right) \beta
$$

and

$$
\gamma_{n 2}=c_{n 20}+k\left(u\left(n^{*}\right), n^{*}-1\right) \alpha+g\left(u\left(n^{*}\right), n^{*}-1\right) \beta .
$$

Now we consider the approximation triangle $\Delta_{n}$ defined by (2.21). We let $\Gamma_{j}$ $(j=0,1,2)$ denote the sides of this triangle which are contained in the straight lines $\Gamma_{n j}\left(x_{1}, x_{2}\right)=0(j=0,1,2$, respectively) defined by (2.20). (See Figure 2, in which the vertices $\mathrm{A}(n, j) / B(n, j)(j=0,1,2)$ are simply labelled by the respective values of $j$ ) We shall show that, at least for large $n$, the slopes of the lines $\Gamma_{n j}\left(x_{1}, x_{2}\right)=0$ depend on the value of $E\left(n^{*}\right)$ in a simple way. Since $\left|E\left(n^{*}\right)\right|$ is bounded by $E_{+}$and $E_{-}$(see (3.8)), this will show that the shape of the triangle $\Delta_{n}$ depends only on the value of $E\left(n^{*}\right)$. Since the shape of $\Delta_{n}$ is what determines $\mu(n+1)$ (see the discussion in Section 2), we will be led to inequalities for $E\left(n^{*}\right)$ like those in Table 5.

We define

$$
s\left(\Gamma_{j}\right)=\text { slope of the line segment } \Gamma_{j} \quad(0 \leqslant j \leqslant 2) .
$$

Thus it follows from (4.5), (4.6), (4.7) and the definition (2.20) of the lines $\Gamma_{n j}\left(x_{1}, x_{2}\right)=0$ that

$$
\begin{aligned}
& s\left(\Gamma_{0}\right)=-k\left(u\left(n^{*}\right)-1, n^{*}\right) / g\left(u\left(n^{*}\right)-1, n^{*}\right), \\
& s\left(\Gamma_{1}\right)=-k\left(u\left(n^{*}\right)+1, n^{*}-1\right) / g\left(u\left(n^{*}\right)+1, n^{*}-1\right), \\
& s\left(\Gamma_{2}\right)=-k\left(u\left(n^{*}\right), n^{*}-1\right) / g\left(u\left(n^{*}\right), n^{*}-1\right) .
\end{aligned}
$$

If we take $m=u\left(n^{*}\right)-1$ and $n=n^{*}$ in (3.13) and divide both sides by $\theta^{\prime \prime} u\left(n^{*}\right) \varphi^{\prime \prime} n^{*}$, then we get

$$
\frac{g(m, n)}{\theta^{\prime \prime} u\left(n^{*}\right) \varphi^{\prime n^{*}}}=\frac{1}{7}\left(\frac{G \theta^{u\left(n^{*}\right)-1} \varphi^{n^{*}}}{\theta^{\prime \prime} u\left(n^{*}\right) \varphi^{\prime \prime n^{*}}}+G^{\prime} E\left(n^{*}\right) \theta^{\prime-1}+G^{\prime \prime} \theta^{\prime \prime-1}\right)
$$

a similar result follows from dividing both sides in (3.14) by $\theta^{\prime \prime} u\left(n^{*}\right) \varphi^{\prime \prime n^{*}}$. Since it follows easily from the definition of $u\left(n^{*}\right)$ that $\left(\theta / \theta^{\prime \prime}\right)^{u\left(n^{*}\right)}\left(\varphi / \varphi^{\prime \prime}\right)^{n^{*}} \rightarrow 0$ as $n^{*} \rightarrow$ 
$-\infty$, we deduce from (4.8) that

$$
s\left(\Gamma_{0}\right) \approx-\frac{K^{\prime} E\left(n^{*}\right) \theta^{\prime-1}+K^{\prime \prime} \theta^{\prime \prime-1}}{G^{\prime} E\left(n^{*}\right) \theta^{\prime-1}+G^{\prime \prime} \theta^{\prime \prime-1}}
$$

for $n^{*}<0,\left|n^{*}\right|$ large (that is, for $n$ large).

We define the function $q_{0}(E)$ by

$$
q_{0}(E)=-\frac{K^{\prime} E \theta^{\prime-1}+K^{\prime \prime} \theta^{\prime \prime-1}}{G^{\prime} E \theta^{\prime-1}+G^{\prime \prime} \theta^{\prime \prime-1}},
$$

so $s\left(\Gamma_{0}\right) \approx q_{0}\left(E\left(n^{*}\right)\right)$ by (4.9). Thus, $s\left(\Gamma_{0}\right)$ depends on $E\left(n^{*}\right)$ in a simple way, as we asserted above (notice since $n^{*}$ is even in class 1 , we have $E\left(n^{*}\right)>0$ so by (3.8) we need only consider $q_{0}(E)$ in the range $2 \theta^{\prime 2}=E_{-}<E<E_{+}=2 \theta^{-1}$ ).

If we further define

$$
q_{1}(E)=-\frac{K^{\prime} E \theta^{\prime} \varphi^{\prime-1}+K^{\prime \prime} \theta^{\prime \prime} \varphi^{\prime \prime-1}}{G^{\prime} E \theta^{\prime} \varphi^{\prime-1}+G^{\prime \prime} \theta^{\prime \prime} \varphi^{\prime \prime-1}}, \quad q_{2}(E)=-\frac{K^{\prime} E \varphi^{\prime-1}+K^{\prime \prime} \varphi^{\prime \prime-1}}{G^{\prime} E \varphi^{\prime-1}+G^{\prime \prime} \varphi^{\prime \prime-1}},
$$

then after proceeding as in the derivation of (4.9) we have

$$
s\left(\Gamma_{j}\right) \approx q_{j}\left(E\left(n^{*}\right)\right) \quad(j=0,1,2) \text { for all large } n .
$$

Calculation of the maxima and minima of the functions $q_{j}(E)$ in the range $E_{-}<E<$ $E_{+}$gives

$$
-1.47<s\left(\Gamma_{0}\right)<-.78, \quad 1.9<s\left(\Gamma_{1}\right)<8.86, \quad-8.86<s\left(\Gamma_{2}\right)<-3.41
$$

Figure 2 is drawn so that the slopes $s\left(\Gamma_{j}\right)$ lie within the ranges given in (4.11); thus the triangle in Figure 2 has a shape which is typical of the shapes of approximation triangles for class 1 (notice that the inequalities (4.11) are restrictive enough to ensure that these approximation triangles are all roughly similar in shape). We are only interested in the ratios of certain lengths determined by the approximation triangles, so only the shape of the triangle in Figure 2 (and not the lengths of its sides) is relevant.

In particular, if we define

$$
\left\|\Gamma_{j}\right\|=\operatorname{span} \text { of } \Gamma_{j} \quad(0 \leqslant j \leqslant 2)
$$

(the definition of span is in Section 2), then $\mu(n+1)$ has the value 2 if and only if

$$
\left\|\Gamma_{2}\right\|\left(\frac{B(n, 1)}{B(n, 0)+B(n, 1)}\right)\left\|\Gamma_{1}\right\|^{-1}<1 .
$$

We derive (4.13) as follows: In obtaining the approximation triangle $\Delta_{n+1}$ from the triangle $\Delta_{n}$ of Figure 2, we insert a new vertex $\mathrm{A}(n+1,1) / B(n+1,1)$ on the side $\Gamma_{2}$ of $\Delta_{n}$. Then $\Delta_{n+1}$ is the triangle with vertices $\mathrm{A}(n, 0) / B(n, 0), \mathbf{A}(n, 2) / B(n, 2)$ (labelled 0 and 2, respectively, in Figure 2), and $\mathbf{A}(n+1,1) / B(n+1,1)$. (See the discussion in Section 2 concerning (2.22).) Also, $\mathbf{A}(n+1, j) / B(n+1, j)=$ $\mathrm{A}(n, j) / B(n, j)$ for $j=0$ and 2 (by (2.6), (2.6b) and (2.6c)). Hence, $\mu(n+1)$ is determined (see the discussion in Section 2) by whichever of the two line segments from $\mathbf{A}(n, 0) / B(n, 0)$ to $\mathbf{A}(n, 2) / B(n, 2)$ and $\mathbf{A}(n+1,1) / B(n+1,1)$, respectively, has the longer span; in fact, $\mu(n+1)=2$ if the former segment is the one with the longer span, and $\mu(n+1)=1$ if the latter segment is the one with the longer span. Now 


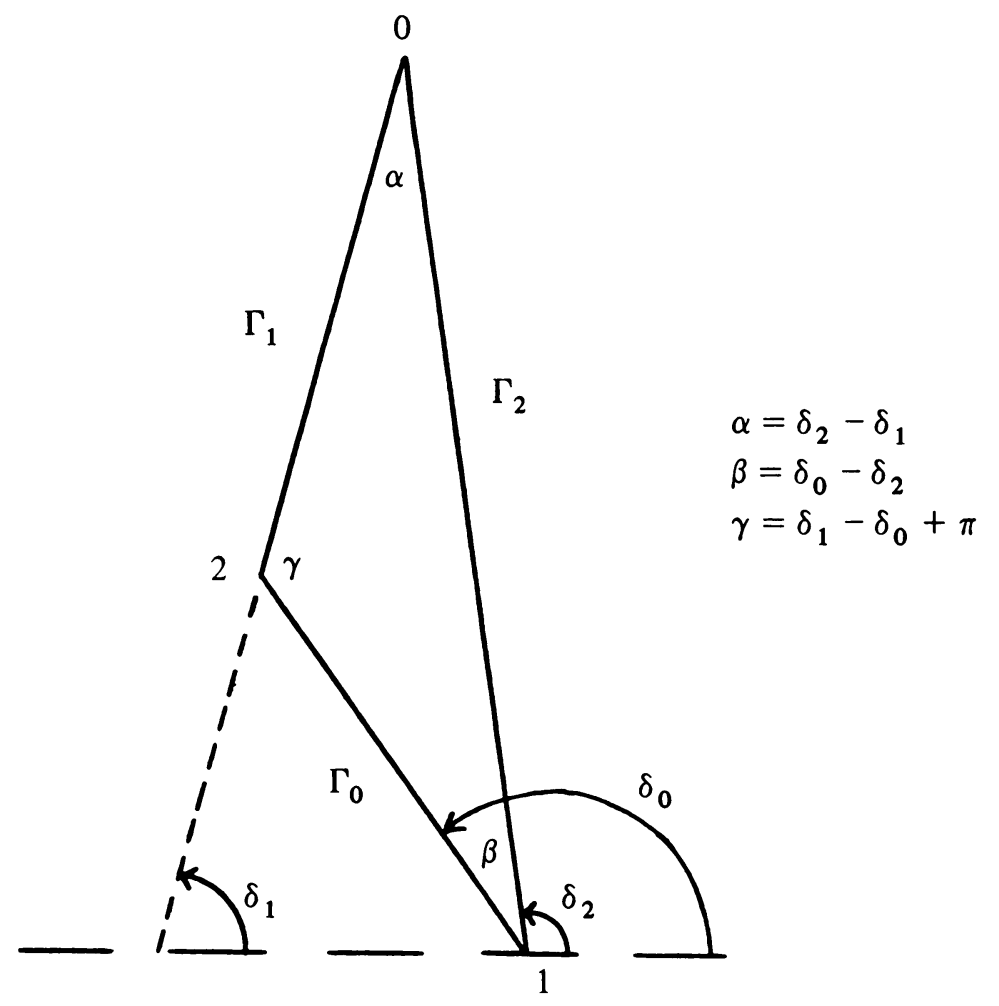

FIGURE 2. Typical approximation triangle, subclass $1 \mathrm{a}$ or $1 \mathrm{~b}$

the span of the former segment is simply $\left\|\Gamma_{1}\right\|$, and it follows from $(2.22)$ with $\mu(n)=$ 1 that the span of the latter segment is $\left\|\Gamma_{2}\right\| B(n, 1) /(B(n, 0)+B(n, 1))$. Thus (4.13) is precisely the condition needed for $\mu(n+1)=2$.

Next we define

$$
\left|\Gamma_{j}\right|=\text { length of } \Gamma_{j} \quad(0 \leqslant j \leqslant 2) .
$$

Of course, $\left|\Gamma_{j}\right|$ is related to the span $\left\|\Gamma_{j}\right\|$ defined in (4.12) by

$$
\left\|\Gamma_{j}\right\|=\max \left(\left|\Gamma_{j} \cos \delta_{j}\right|,\left|\Gamma_{j}\right| \sin \delta_{j}\right),
$$

where $\delta_{j}\left(0 \leqslant \delta_{j}<\pi\right)$ is the angle between the $x_{1}$-axis and the line $\Gamma_{n j}\left(x_{1}, x_{2}\right)=0$ (see Figure 2).

Since by (4.11) we have $\left|s\left(\Gamma_{1}\right)\right|>1$ and $\left|s\left(\Gamma_{2}\right)\right|>1$, it follows from Figure 2 and (4.14) that $\left\|\Gamma_{j}\right\|=\left|\Gamma_{j}\right| \sin \delta_{j}$ for $j=1,2$. Using Figure 2 and some trigonometry, we have

$$
\left|\Gamma_{2}\right|\left|\Gamma_{1}\right|^{-1}=\sin \gamma / \sin \beta=\frac{\sin \delta_{0} \cos \delta_{1}-\sin \delta_{1} \cos \delta_{0}}{\sin \delta_{0} \cos \delta_{2}-\sin \delta_{2} \cos \delta_{0}}
$$

and therefore,

$$
\left\|\Gamma_{2}\right\|\left\|\Gamma_{1}\right\|^{-1}=\frac{\tan \delta_{2}\left(\tan \delta_{0}-\tan \delta_{1}\right)}{\tan \delta_{1}\left(\tan \delta_{0}-\tan \delta_{2}\right)} .
$$

Since $\tan \delta_{j}=s\left(\Gamma_{j}\right)(0 \leqslant j \leqslant 2),(4.10)$ gives 


$$
\left\|\Gamma_{2}\right\|\left\|\Gamma_{1}\right\|^{-1} \approx \frac{q_{2}\left(q_{0}-q_{1}\right)}{q_{1}\left(q_{0}-q_{2}\right)} \quad\left(q_{j}=q_{j}\left(E\left(n^{*}\right)\right)\right) .
$$

Also, it follows from Lemma $1,(2.15)$ and $(4.5)$ to (4.7) that

$$
\frac{B(n, 0)}{B(n, 1)}=\left|\frac{c_{n 11} c_{n 22}-c_{n 21} c_{n 12}}{c_{n 21} c_{n 02}-c_{n 01} c_{n 22}}\right|
$$

$$
=\left|\frac{k\left(u\left(n^{*}\right)+1, n^{*}-1\right) g\left(u\left(n^{*}\right), n^{*}-1\right)-k\left(u\left(n^{*}\right), n^{*}-1\right) g\left(u\left(n^{*}\right)+1, n^{*}-1\right)}{k\left(u\left(n^{*}\right), n^{*}-1\right) g\left(u\left(n^{*}\right)-1, n^{*}\right)-k\left(u\left(n^{*}\right)-1, n^{*}\right) g\left(u\left(n^{*}\right), n^{*}-1\right)}\right| .
$$

If the method of deriving (4.9) is applied to the right-hand side of (4.16), we find that $B(n, 0) / B(n, 1)$ is approximately equal to a certain function of $E\left(n^{*}\right)$ for $n^{*}<0,\left|n^{*}\right|$ large. Calculation of this function shows that it is in fact a constant, namely, $-\theta^{\prime \prime}-1$. Thus, we have proved that

$$
\lim _{n \rightarrow \infty} B(n, 0) / B(n, 1)=-\theta^{\prime \prime}-1 \approx .8019 .
$$

Now let $\Gamma\left(E\left(n^{*}\right)\right)$ denote the function on the right-hand side of (4.15). Calculation shows that for $E_{-}<E\left(n^{*}\right)<E_{+}$, the function $\Gamma\left(E\left(n^{*}\right)\right)$ is monotone increasing and

$$
\Gamma\left(E\left(n^{*}\right)\right) \leqslant-\theta^{\prime \prime} \quad \text { if and only if } E\left(n^{*}\right) \leqslant \theta^{4}\left|\theta^{\prime}\right| .
$$

Combining (4.15), (4.17) and (4.18), we may conclude that (4.13) holds if and only if $E_{-}<E\left(n^{*}\right)<\theta^{4}\left|\theta^{\prime}\right|$. This means that for $\tau_{n}$ in subclass $1 \mathrm{a}$ or $1 \mathrm{~b}$, we have $\tau_{n+1}$ in class 7 only if $E_{-}<E\left(n^{*}\right)<\theta^{4}\left|\theta^{\prime}\right|$, and otherwise $\tau_{n+1}$ is in class 5 . This proves part of the restrictions on $E\left(n^{*}\right)$ stated in Table 5 for the subclasses of class 1 . The remainder of the restrictions (in particular, the range of $E\left(n^{*}\right)$ in which subclass $1 \mathrm{c}$ occurs) will follow from the proof of Theorem 5 below.

We pause for a digression at this point. To avoid needless complication of the proof of Theorem 4, nothing has so far been said about the accuracy of the approximate equalities in (4.10) and (4.15) (although it does of course follow from the work above that the difference between the two sides of these equalities $\rightarrow 0$ as $n^{*} \rightarrow-\infty$ ). This does not affect the proof, except for the following: the possibility that for $E\left(n^{*}\right)$ less than but very near to $\theta^{4}\left|\theta^{\prime}\right| \approx 1.0761$ we could have (4.13) false despite the fact that (4.18) is true (because the approximation (4.15) is used in deducing (4.13) from (4.18)) has not been ruled out. In fact, this possibility cannot occur, because the work of Baker (for example, the theorem in [1]) provides a lower bound for $\left|E\left(n^{*}\right)-\theta^{4}\right| \theta^{\prime}||$ of the form $c(1) /\left(n^{*}\right)^{c(2)}(c(1)$ and $c(2)$ computable constants), and this lower bound implies that $E\left(n^{*}\right)$ cannot approach $\theta^{4}\left|\theta^{\prime}\right|$ quickly enough for the unwanted possibility to occur. The detailed justification of this is straightforward, so we omit it. Appeals to the result of Baker [1] are needed at various other places in our proofs, but in every case the application of the theorem of Baker is very similar to the one we have just explained; thus, from now on we shall not explicitly point out the places where we need to use this theorem. 


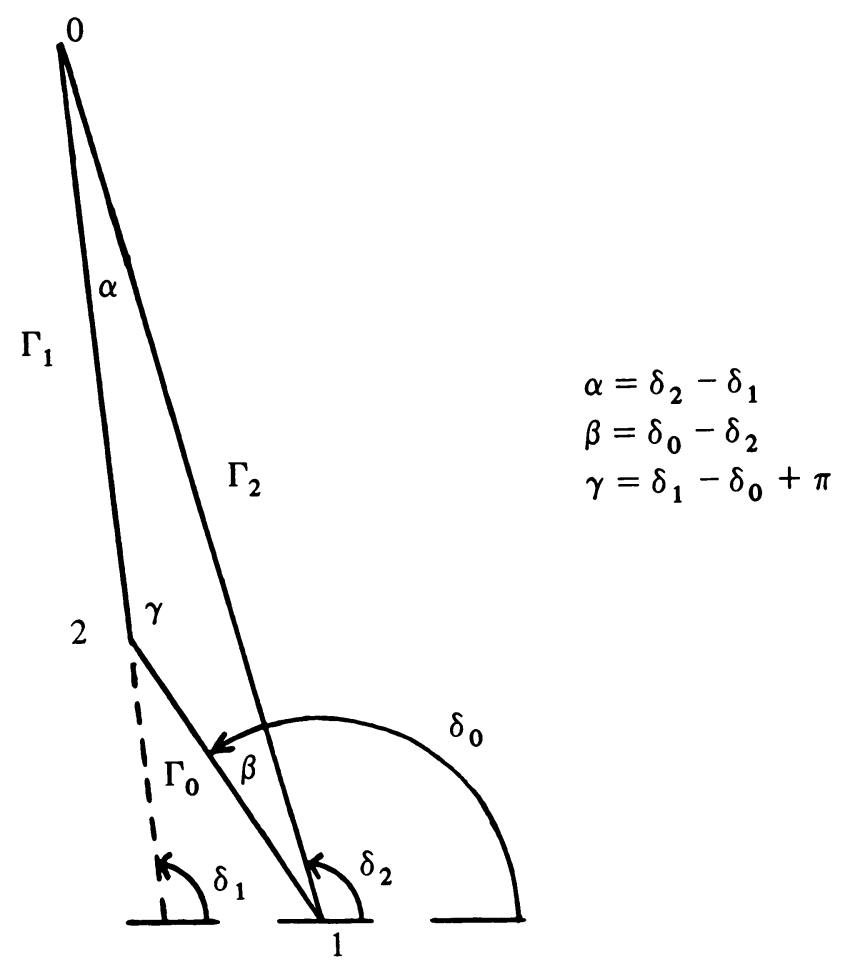

FIGURE 3. Typical approximation triangle, subclass 1c

Now we finish the calculations for class 1 ; we assume that $\tau_{n}$ is in subclass $1 \mathrm{c}$, so $n^{*}$ is even and the index of $\gamma_{n 0}$ is -2 . Thus, we obtain the following analogues of (4.5), (4.6) and (4.7):

$$
\begin{aligned}
& \gamma_{n 0}=c_{n 0}+k\left(u\left(n^{*}\right)-2, n^{*}\right) \alpha+g\left(u\left(n^{*}\right)-2, n^{*}\right) \beta \\
& \gamma_{n 1}=c_{n 10}+k\left(u\left(n^{*}\right), n^{*}-1\right) \alpha+g\left(u\left(n^{*}\right), n^{*}-1\right) \beta \\
& \gamma_{n 2}=c_{n 20}+k\left(u\left(n^{*}\right)-1, n^{*}-1\right) \alpha+g\left(u\left(n^{*}\right)-1, n^{*}-1\right) \beta .
\end{aligned}
$$

As before, we consider the approximation triangle $\Delta_{n}$, and we use (4.19) to determine the slopes $s\left(\Gamma_{j}\right)(0 \leqslant j \leqslant 2)$ of the sides of the triangle (compare (4.8)). Calculations like those used to derive (4.9) give

$$
s\left(\Gamma_{j}\right) \approx r_{j}\left(E\left(n^{*}\right)\right) \quad(j=0,1,2) \text { for all large } n,
$$

where

$$
\begin{aligned}
& r_{0}(E)=-\frac{K^{\prime} E \theta^{\prime-2}+K^{\prime \prime} \theta^{\prime \prime-2}}{G^{\prime} E \theta^{\prime-2}+G^{\prime \prime} \theta^{\prime \prime-2}} \\
& r_{j}(E)=-\frac{K^{\prime} E \theta^{\prime 1-j} \varphi^{\prime-1}+K^{\prime \prime} \theta^{\prime 1-j} \varphi^{\prime \prime-1}}{G^{\prime} E \theta^{\prime-j} \varphi^{\prime-1}+G^{\prime \prime} \theta^{\prime 1-j} \varphi^{\prime \prime-1}} \quad(j=1,2) .
\end{aligned}
$$

Calculation of the maxima and minima of the functions $r_{j}(E)$ in the range $E_{-}<E<$ $E_{+}$gives 
(4.21) $-1.72<s\left(\Gamma_{0}\right)<-1.46, \quad-8.86<s\left(\Gamma_{1}\right)<-3.41, \quad-3.42<s\left(\Gamma_{2}\right)<-2.08$.

The triangle in Figure 3 is drawn so that the slopes $s\left(\Gamma_{j}\right)$ lie within the ranges given in (4.21).

Just as in the subclasses $1 \mathrm{a}$ and $1 \mathrm{~b}$, we have $\mu(n+1)=2$ if and only if (4.13) holds. Since by (4.21) we have $\left|s\left(\Gamma_{1}\right)\right|>1$ and $\left|s\left(\Gamma_{2}\right)\right|>1$, the same reasoning by which we deduced (4.15) from Figure 2 and (4.10) leads us to deduce

$$
\left\|\Gamma_{2}\right\|\left\|\Gamma_{1}\right\|^{-1} \approx \frac{r_{2}\left(r_{0}-r_{1}\right)}{r_{1}\left(r_{0}-r_{2}\right)} \quad\left(r_{j}=r_{j}\left(E\left(n^{*}\right)\right)\right)
$$

from Figure 3 and (4.20). Following the derivation of (4.17) leads to

$$
\lim _{n \rightarrow \infty} B(n, 0) / B(n, 1)=-\theta^{\prime \prime}-1 \approx .8019 .
$$

Now let $\Gamma\left(E\left(n^{*}\right)\right)$ denote the function on the right-hand side of (4.22). Calculation shows that for $E_{-}<E\left(n^{*}\right)<E_{+}$, the function $\Gamma\left(E\left(n^{*}\right)\right)$ is monotone increasing and $\Gamma\left(E\left(n^{*}\right)\right)>1.9$ always. Combining this inequality with (4.23), we conclude that (4.13) is always false. Hence $\mu(n+1)=1$ always holds for $\tau_{n}$ in subclass $1 \mathrm{c}$, and this means $\tau_{n+1}$ is always in class 5 , as specified in Table 5 . We shall see below (in the proof of Theorem 5) that in fact subclass $1 \mathrm{c}$ only occurs for the restricted range of $E\left(n^{*}\right)$ given in Table 5 .

Calculations for Class 3. Since $\tau_{n}$ is in class 3 , we have $\epsilon(n+1)=\mu(n)=1$ from Table 3. Hence by (2.14) to (2.14b) and the rest of the data for class 3 in Table 3 , we have

$$
\gamma_{n+1,0}=\theta^{m(1)-1}|\varphi|^{m(2)+1}-\theta^{m(1)}|\varphi|^{m(2)}, \quad \gamma_{n+1,1}=\gamma_{n 0}, \gamma_{n+1,2}=\gamma_{n 2},
$$

with $m(1)$ and $m(2)$ determined by (4.3). Thus, using the identity $\theta^{-1}|\varphi|-1=-\theta^{\prime \prime}-$ $1=\theta^{-1}$, we get

$$
\begin{gathered}
\gamma_{n+1,0}=\theta^{m(1)-1}|\varphi|^{m(2)}, \quad \gamma_{n+1,1}=\theta^{m(1)}|\varphi|^{m(2)} \\
\gamma_{n+1,2}=\theta^{m(1)}|\varphi|^{m(2)+1}
\end{gathered}
$$

It follows from (2.13) and (4.24) that $\epsilon(n+2)=1$, so the Eqs. (4.24) and Table 3 imply that $\tau_{n+1}$ is in class 6 if and only if $\mu(n+1)=2$. We shall prove that $\mu(n+1)$ $=2$ always holds if $\tau_{n}$ is in class 3 .

We first assume that $\tau_{n}$ is in subclass 3 a so $n^{*}$ is odd and the index of $\gamma_{n 0}$ is 0 .

Thus we obtain (using (4.3) with $i=0$ ) the following analogues of (4.5), (4.6) and (4.7):

$$
\begin{aligned}
& \gamma_{n 0}=c_{n 0}+k\left(u\left(n^{*}\right), n^{*}\right) \alpha+g\left(u\left(n^{*}\right), n^{*}\right) \beta, \\
& \gamma_{n 1}=c_{n 10}+k\left(u\left(n^{*}\right)-1, n^{*}+1\right) \alpha+g\left(u\left(n^{*}\right)-1, n^{*}+1\right) \beta, \\
& \gamma_{n 2}=c_{n 20}+k\left(u\left(n^{*}\right), n^{*}+1\right) \alpha+g\left(u\left(n^{*}\right), n^{*}+1\right) \beta .
\end{aligned}
$$

As before, we consider the approximation triangle $\Delta_{n}$, and we use (4.25) to determine the slopes $s\left(\Gamma_{j}\right)(0 \leqslant j \leqslant 2)$ of the sides of the triangle. Calculations like those used to derive (4.9) give

$$
s\left(\Gamma_{j}\right) \approx t_{j}\left(E\left(n^{*}\right)\right) \quad(j=0,1,2) \text { for all large } n,
$$


where

$$
t_{0}(E)=-\frac{E K^{\prime}+K^{\prime \prime}}{E G^{\prime}+G^{\prime \prime}}, \quad t_{j}(E)=-\frac{K^{\prime} E \theta^{\prime j-2} \varphi^{\prime}+K^{\prime \prime} \theta^{\prime \prime j-2} \varphi^{\prime \prime}}{G^{\prime} E \theta^{\prime j-2} \varphi^{\prime}+G^{\prime \prime} \theta^{\prime \prime j-2} \varphi^{\prime \prime}} \quad(j=1,2) .
$$

Calculation of the maxima and minima of the functions $t_{j}(E)$ in the range $-E_{+}<E<$ $-E_{-}$(because $n^{*}$ is odd, $E\left(n^{*}\right)<0$ ) gives

$$
\begin{array}{ll}
-\infty<s\left(\Gamma_{0}\right)<-4.85 & \text { if }-E_{+}<E\left(n^{*}\right)<-\theta^{-1}, \\
4.22<s\left(\Gamma_{0}\right)<+\infty & \text { if }-\theta^{-1}<E\left(n^{*}\right)<-E_{-}
\end{array}
$$

(this follows since $E G^{\prime}+G^{\prime \prime}=0$ for $E=-G^{\prime \prime} / G^{\prime}=-\theta^{-1} \approx-.8019$ ) and

$$
-1.35<s\left(\Gamma_{1}\right)<-.52, \quad-.53<s\left(\Gamma_{2}\right)<.48
$$

The triangle in Figure 4 is drawn so that the slopes $s\left(\Gamma_{j}\right)$ are about what they would be for $E\left(n^{*}\right) \approx-E_{-}$; namely, $s\left(\Gamma_{0}\right) \approx 4.22, s\left(\Gamma_{1}\right) \approx-.52, s\left(\Gamma_{2}\right) \approx .47$. As $E\left(n^{*}\right)$ decreases from $-E_{-}$, the shape of the triangle $\Delta_{n}$ tends to an isosceles right triangle, which is the shape of the triangle for $E\left(n^{*}\right) \approx-\theta^{-1}$. (For this value of $E\left(n^{*}\right)$ we have $s\left(\Gamma_{0}\right) \approx \infty, s\left(\Gamma_{1}\right) \approx-1, s\left(\Gamma_{2}\right) \approx 0$, so the hypotenuse of the right triangle would be the line segment from 0 to 2 in Figure 4.) It is evident from their shapes that for any of these triangles $\Delta_{n}$ with $-\theta^{-1}<E\left(n^{*}\right)<-E_{-}$, we have $\mu(n)=1$, as we must have in class 3. However, for $-E_{+}<E\left(n^{*}\right)<-\theta^{-1}$ the shape of the triangle $\Delta_{n}$ would be such that $\mu(n)=2$ (contradicting our assumption that $\tau_{n}$ is in class $3 \mathrm{a}$ ). Thus, our assumption that $\tau_{n}$ is in class 3 a implies that $E_{-}<-E\left(n^{*}\right)<\theta^{-1}$, which is exactly the restriction on $E\left(n^{*}\right)$ listed for class $3 \mathrm{a}$ in Table 5. Some (though not all) of the

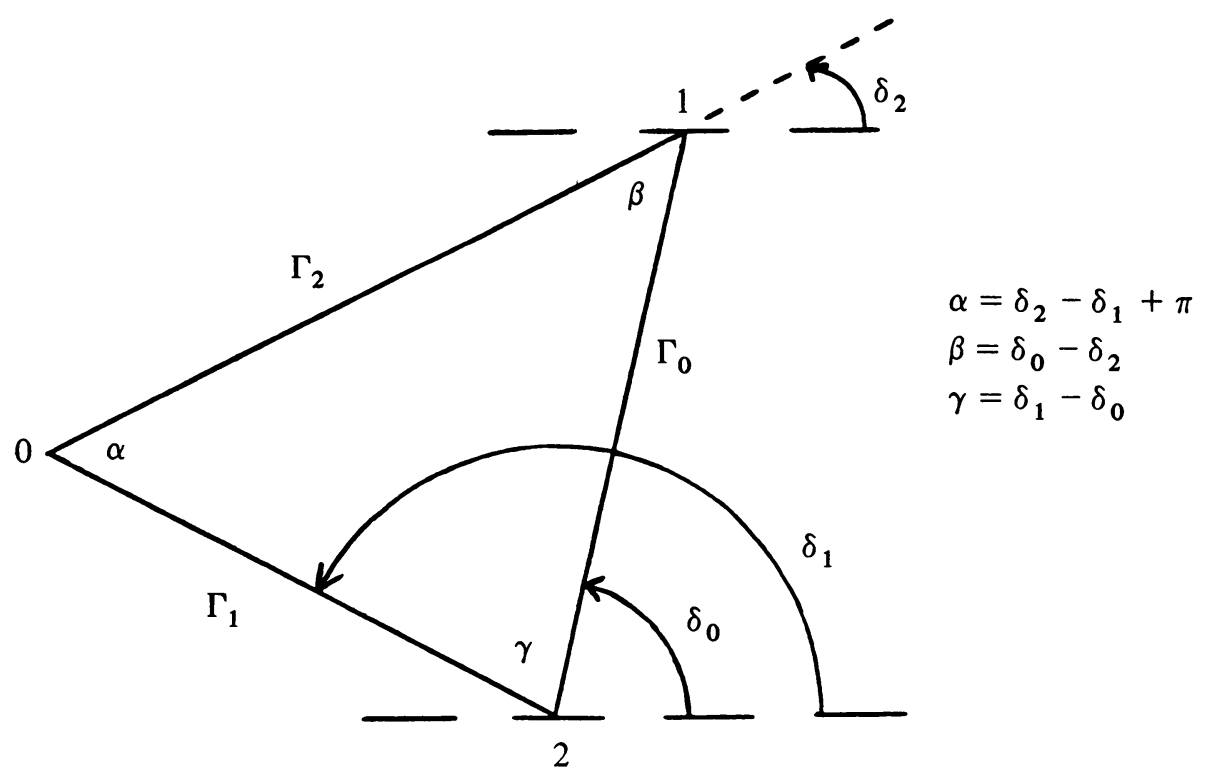

FIGURE 4. Typical approximation triangle, subclass 3a

other restrictions on $E\left(n^{*}\right)$ listed in Table 5 can be deduced in a similar way. A full derivation of all the restrictions on $E\left(n^{*}\right)$ listed in Table 5 must wait until the proof of Theorem 5 below. 


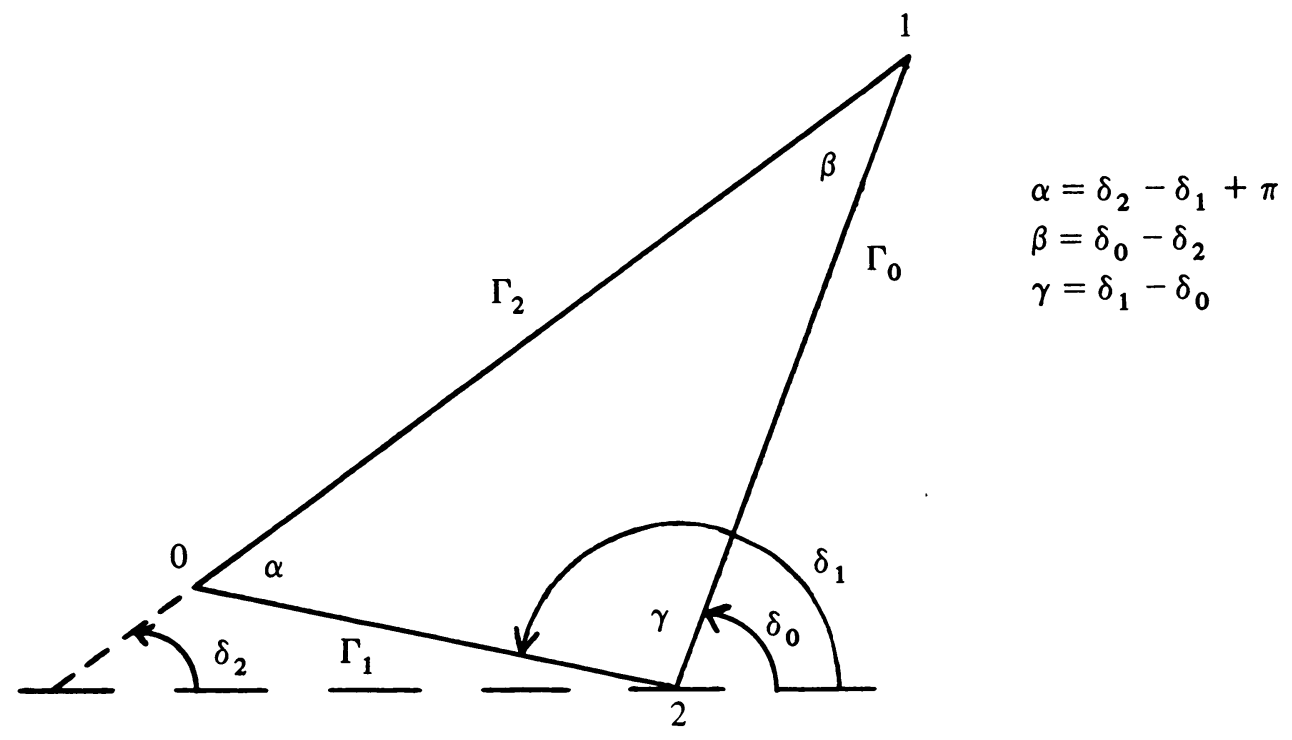

FIgURE 5. Typical approximation triangle, subclass $3 \mathrm{~b}$

Proceeding as in the derivation of (4.13) (except that now $\Delta_{n+1}$ is the triangle with vertices $\mathbf{A}(n, 1) / B(n, 1), \mathbf{A}(n, 2) / B(n, 2)$ and $\mathbf{A}(n+1,1) / B(n+1,1)$, as in Figure 1 ; and we have $\epsilon(n+1)=\mu(n)=1$ in (2.6) to (2.6c), since $\tau_{n}$ is in class 3$)$, we find that $\mu(n+1)$ is determined by whichever of the two line segments from $\mathrm{A}(n, 1) / B(n, 1)$ to $\mathbf{A}(n, 2) / B(n, 2)$ and $\mathbf{A}(n+1,1) / B(n+1,1)$, respectively, has the longer span.

Thus $\mu(n+1)=2$ if and only if

$$
\left\|\Gamma_{2}\right\|\left(\frac{B(n, 0)}{B(n, 0)+B(n, 1)}\right)\left\|\Gamma_{0}\right\|^{-1}<1
$$

We have $\left|s\left(\Gamma_{0}\right)\right|>1$ and $\left|s\left(\Gamma_{2}\right)\right|<1$ by (4.28). Thus $\left\|\Gamma_{0}\right\|=\left|\Gamma_{0}\right| \sin \delta_{0}$ and $\left\|\Gamma_{2}\right\|=\left|\Gamma_{2} \cos \delta_{2}\right|$. Using Figure 4 and some trigonometry, we have

$$
\left|\Gamma_{2}\right|\left|\Gamma_{0}\right|^{-1}=\sin \gamma / \sin \alpha=\frac{\sin \delta_{1} \cos \delta_{0}-\sin \delta_{0} \cos \delta_{1}}{\sin \delta_{1} \cos \delta_{2}-\sin \delta_{2} \cos \delta_{1}} ;
$$

and therefore $\left(\left\|\Gamma_{2}\right\|=+\left|\Gamma_{2}\right| \cos \delta_{2}\right.$ since $0<\delta_{2}<\pi / 2$ in Figure 4)

$$
\left\|\Gamma_{2}\right\|\left\|\Gamma_{0}\right\|^{-1}=\frac{1}{\tan \delta_{0}} \cdot \frac{\tan \delta_{1}-\tan \delta_{0}}{\tan \delta_{1}-\tan \delta_{2}} .
$$

Since $\tan \delta_{j}=s\left(\Gamma_{j}\right)(0 \leqslant j \leqslant 2),(4.26)$ gives

$$
\left\|\Gamma_{2}\right\|\left\|\Gamma_{0}\right\|^{-1} \approx \frac{t_{1}-t_{0}}{t_{0}\left(t_{1}-t_{2}\right)} \quad\left(t_{j}=t_{j}\left(E\left(n^{*}\right)\right)\right) .
$$

Following the derivation of (4.17) leads to

$$
\lim _{n \rightarrow \infty} B(n, 1) / B(n, 0)=-\theta^{\prime \prime} \approx 1.8019 .
$$

Now let $\Gamma\left(E\left(n^{*}\right)\right)$ denote the function on the right-hand side of (4.30). Calculation shows that for $-E_{+}<E\left(n^{*}\right)<-E_{-}$, the function $\Gamma\left(E\left(n^{*}\right)\right)$ is monotone decreas- 
ing and $\Gamma\left(E\left(n^{*}\right)\right)<1.2$ always. Combining this inequality with (4.31), we conclude that (4.29) is always true. Hence $\mu(n+1)=2$ always holds for $\tau_{n}$ in subclass $3 \mathrm{a}$, and this means $\tau_{n+1}$ is always in class 6 , as specified in Table 5 .

Now we assume that $\tau_{n}$ is in subclass $3 b$, so $n^{*}$ is odd and the index of $\gamma_{n 0}$ is 1 . Thus we obtain the following analogue of (4.25):

$$
\begin{aligned}
& \gamma_{n 0}=c_{n 0}+k\left(u\left(n^{*}\right)+1, n^{*}\right) \alpha+g\left(u\left(n^{*}\right)+1, n^{*}\right) \beta, \\
& \gamma_{n 1}=c_{n 10}+k\left(u\left(n^{*}\right), n^{*}+1\right) \alpha+g\left(u\left(n^{*}\right), n^{*}+1\right) \beta \\
& \gamma_{n 2}=c_{n 20}+k\left(u\left(n^{*}\right)+1, n^{*}+1\right) \alpha+g\left(u\left(n^{*}\right)+1, n^{*}+1\right) \beta .
\end{aligned}
$$

As before, we consider the approximation triangle $\Delta_{n}$, and we use (4.32) to determine the slopes $s\left(\Gamma_{j}\right)(0 \leqslant j \leqslant 2)$ of the sides of the triangle. Calculations like those used to derive (4.9) give

$$
s\left(\Gamma_{j}\right) \approx w_{j}\left(E\left(n^{*}\right)\right) \quad(j=0,1,2) \text { for all large } n,
$$

where

$$
w_{0}(E)=-\frac{E K^{\prime} \theta^{\prime}+K^{\prime \prime} \theta^{\prime \prime}}{E G^{\prime} \theta^{\prime}+G^{\prime \prime} \theta^{\prime \prime}}, \quad w_{j}(E)=-\frac{K^{\prime} E \theta^{\prime j-1} \varphi^{\prime}+K^{\prime \prime} \theta^{\prime \prime j-1} \varphi^{\prime \prime}}{G^{\prime} E \theta^{\prime j-1} \varphi^{\prime}+G^{\prime \prime} \theta^{\prime \prime j-1} \varphi^{\prime \prime}} \quad(j=1,2) .
$$

Calculation of the maxima and minima of the functions $w_{j}(E)$ in the range $-E_{+}<E<$ $-E_{-}$gives

$$
1.67<s\left(\Gamma_{0}\right)<4.23, \quad-.53<s\left(\Gamma_{1}\right)<.48, .47<s\left(\Gamma_{2}\right)<1.01 .
$$

The triangle in Figure 5 is drawn so that the slopes $s\left(\Gamma_{j}\right)$ lie within the ranges given in (4.34).

Just as in subclass $3 \mathrm{a}$, we have $\mu(n+1)=2$ if and only if (4.29) holds. We have $\left|s\left(\Gamma_{0}\right)\right|>1$ by $(4.34)$ and $\left|s\left(\Gamma_{2}\right)\right|<1$ by (4.34) and the restriction $E\left(n^{*}\right)>-.8271$ given for subclass $3 b$ in Table 5 (since $s\left(\Gamma_{2}\right) \approx 1.01$ when $E\left(n^{*}\right) \approx-E_{-}$, and this value of $E\left(n^{*}\right)$ is excluded by Table 5; as a matter of fact, allowing $E\left(n^{*}\right) \approx-E_{-}$, so that $\left|s\left(\Gamma_{2}\right)\right|>1$, would not change the results of our calculations, but would introduce a superfluous computation). Hence the same reasoning by which we deduced (4.30) from Figure 4 and (4.26) leads us to deduce

$$
\left\|\Gamma_{2}\right\|\left\|\Gamma_{0}\right\|^{-1} \approx \frac{w_{0}-w_{1}}{w_{0}\left(w_{2}-w_{1}\right)} \quad\left(w_{j}=w_{j}\left(E\left(n^{*}\right)\right)\right)
$$

from Figure 5 and (4.33). Following the derivation of (4.17) leads to

$$
\lim _{n \rightarrow \infty} B(n, 1) / B(n, 0)=-\theta^{\prime \prime} \approx 1.8019 .
$$

Now let $\Gamma\left(E\left(n^{*}\right)\right)$ denote the function on the right-hand side of (4.35). Calculation shows that for $-E_{+}<E\left(n^{*}\right)<-E_{-}$, the function $\Gamma\left(E\left(n^{*}\right)\right)$ is monotone increasing and $\Gamma\left(E\left(n^{*}\right)\right)<1.35$ always. Combining this inequality with (4.31) we conclude that (4.29) is always true. Hence $\mu(n+1)=2$ always holds for $\tau_{n}$ in subclass $3 \mathrm{~b}$, and this means $\tau_{n+1}$ is always in class 6 , as specified in Table 5 . We shall see below (in the proof of Theorem 5) that in fact subclass $3 b$ only occurs for the restricted range of $E\left(n^{*}\right)$ given in Table 5 . 
Patterns A. B, C1

\begin{tabular}{|c|c|c|c|c|c|}
\hline $7_{9}^{*}$ & $3_{6}$ & $8_{5}$ & & & \\
\hline${ }^{*} 4$ & ${ }^{*}{ }^{0}$ & $5_{4}^{*}$ & $\begin{array}{c}* \\
3_{1}\end{array}$ & $7_{-2}^{* k}$ & $3_{-5}$ \\
\hline $1_{14}$ & $2_{11}$ & $I_{8}$ & $b_{2}$ & ${ }^{*}{ }^{0}$ & $\begin{array}{c}* \\
6 \text { * * } \\
6_{-4}\end{array}$ \\
\hline & & & & $I_{3}$ & $\alpha_{0}$ \\
\hline $\mathrm{n}^{*}-5$ & $n^{*}-4$ & $\mathrm{n}^{*}-3$ & $n^{*}-2$ & $n^{*}-1$ & $n^{*}$ \\
\hline
\end{tabular}

$\begin{array}{cccc}j & \begin{array}{c}\text { C1as8 of } \\ \tau_{n+j}\end{array} & \epsilon(n+j+1) & \mu(n+j) \\ 0 & 2 & 0 & 2 \\ 1 & 3 & 1 & 1 \\ 2 & 6 & 1 & 2 \\ 3 & 1 & 0 & 1 \\ 4 & 5 & 1 & 1 \\ 5 & 8 & 1 & 2 \\ 6 & 3 & 1 & 1 \\ 7 & 6 & 1 & 2 \\ 8 & 1 & 0 & 1 \\ 9 & 7 & 1 & 2 \\ 10 & 4 & 1 & 1\end{array}$

The position of $u(1)\left(n^{*}+5 \leq 1 \leq n^{*}\right)$ is indicated as follors: pattern A,* ; pattern $\mathrm{B}, \bullet$ : pattern $\mathrm{Cl}$,

Patterns C2, D

$$
\int \frac{\text { Class of }}{\tau_{n+j}} \in(n+1+1) \mu(n+j)
$$

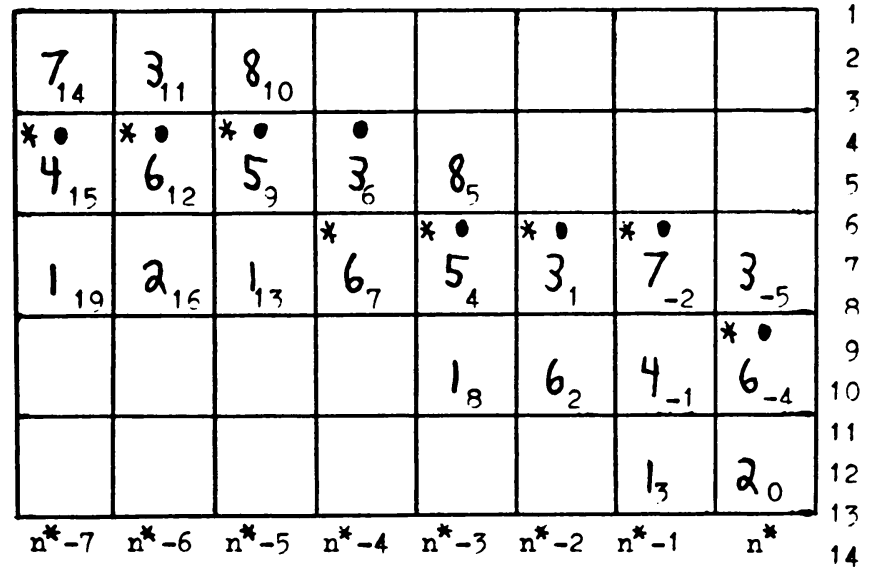

$\begin{array}{lll}2 & 0 & 2 \\ 3 & 1 & 1 \\ 6 & 1 & 2 \\ 1 & 0 & 1 \\ 5 & 1 & 1 \\ 8 & 1 & 2 \\ 3 & 1 & 1 \\ 6 & 1 & 2 \\ 1 & 0 & 1 \\ 5 & 1 & 1 \\ 8 & 1 & 2 \\ 3 & 1 & 1 \\ 6 & 1 & 2 \\ 1 & 0 & 1 \\ 7 & 1 & 2 \\ 4 & 1 & 1\end{array}$

The nosition of $u(1)\left(n^{*}-7 \leq 1 \leq n^{*}\right)$ is indicated 19 as follows: pattern C2,*; pattern $D, \bullet$.

The position of the first component $\gamma_{n+1,0}$ of each triple $\tau_{n+f}$ in the $s(m, n)$ arrar is indicated br the class label of $\tau_{n+f}$ with a subscript $f$ (e. $x_{0}$, the position of $\gamma_{n 0}$ is indicated by $2_{0}$ since $\tau_{n}$ is in class 2$)$. Subscripts $f<0$ Indicate components $\gamma_{n+j, 0}(j<0)$ of triples $\tau_{n+j}$ in the nrevious pattern. Subseriots $f>10$ for patterns A. B. C1 or $f>15$ for patterns C2, D indicate components of triples $\tau_{n+f}$ in the next pattern. The integer $n^{*}<0$ is always odd.

FIGURE 6. Arrangement of patterns of triples $\tau_{n+j}(0 \leqslant j \leqslant 10$ or $0 \leqslant j \leqslant 15)$ in the $S(m, n)$ array

The proof of Theorem 4 is complete once the calculations for all ten classes are carried out. Of course, it is assumed in making these calculations that the finite list of subclasses in Table 5 is complete, and that the indices, parity of $n^{*}$ and restrictions on $E\left(n^{*}\right)$ listed there are correct. Our next theorem justifies all of the entries in Table 5 , and provides some information about the sequence of class labels for the triples $\tau_{n}$ (this sequence for $4 \leqslant n \leqslant 38$ is given in Table 2 ). 
Before we can state Theorem 5, we need some more definitions. We define patterns $\mathrm{A}, \mathrm{B}, \mathrm{C}, \mathrm{D}$ of values of $u(n), n<0$, as follows:

Pattern A-u(n) $=u(n-1), u(n-2)=u(n-1)+1=u(n-j)(j=3,4,5)$.

Pattern B-u(n)=u(n-1), $u(n-2)=u(n-1)+1=u(n-j)(j=3,4)$,

$$
u(n-5)=u(n-4)+1 \text {. }
$$

Pattern C-u(n-1) $=u(n)+1=u(n-j)(j=2,3,4), u(n-5)=u(n-4)+1$.

Pattern D-u(n-1)=u(n)+1=u(n-j) $(j=2,3), u(n-4)=u(n-3)+1=$ $u(n-5)$.

By Lemma 4 , these are the only possible patterns of values of $u(n-j), 0 \leqslant j \leqslant 5$, if $u(n-2)>u(n)$.

It turns out that whenever $\tau_{n}$ is in class 2 , we have $u\left(n^{*}-2\right)>u\left(n^{*}\right)$ for the associated integer $n^{*}$; thus for these values of $n^{*}$ the pattern of values of $u\left(n^{*}\right)$ is always one of the patterns A, B, C, D given above. Furthermore, it turns out that for $n \geqslant 17$, whenever $\tau_{n}$ is in class 2 the sequence of class labels for $\tau_{n+j}$ is determined for $0 \leqslant$ $j \leqslant 10$ or for $0 \leqslant j \leqslant 15$. In fact, we either have

$$
\tau_{n}, \tau_{n+1}, \ldots, \tau_{n+10} \text { are in classes } 2,3,6,1,5,8,3,6,1,7,4 \text {, respectively, }
$$

or

$$
\tau_{n}, \tau_{n+1}, \ldots, \tau_{n+15}
$$

are in classes $2,3,6,1,5,8,3,6,1,5,8,3,6,1,7,4$, respectively.

Also, whenever $\tau_{n}(n \geqslant 17)$ is in class 2 , the index of $\gamma_{n 0}$ is -1 ; hence (4.37) or (4.38), in conjunction with the last column of Table 4 , determines the indices of $\gamma_{n+j, 0}$ and the associated integers $(n+j) *$ for each $j, 0 \leqslant j \leqslant 10$ or $0 \leqslant j \leqslant 15$, respectively (see Figure 6). All the statements in this paragraph (plus more) are proved in Theorem 5.

THEOREM 5. Suppose $n \geqslant 17$. Then whenever $\tau_{n}$ is in class 2 , it follows that $n^{*}$ is odd, $u\left(n^{*}-2\right)>u\left(n^{*}\right)$ and the index of $\gamma_{n 0}$ is -1 . Also, either the classes of $\tau_{j}$ and the values of $j^{*}$ and of the index of $\gamma_{j 0}$ are determined for $n \leqslant j \leqslant n+10$ by one of the patterns A, B, C1 of Figure 6; or the classes of $\tau_{j}$ and the values of $j^{*}$ and of the index of $\gamma_{j 0}$ are determined for $n \leqslant j \leqslant n+15$ by one of the patterns C2, D of Figure 6. This gives the correct values for the parity of $n^{*}$ in Table 5, and shows that the finite list of indices there is complete. Further, each entry in one of the patterns of Figure 6 only occurs when the restrictions given in Table 6 for the corresponding value of $E\left(n^{*}\right)$ are satisfied. This gives the restrictions on $E\left(n^{*}\right)$ given in Table 5. Finally, each triple $\tau_{j}$ is in one of the patterns A, B, C1, C2 or D of Figure 6; that is, the sequence $\tau_{n}, n \geqslant 17$, can be divided into a sequence of blocks (with 11 or 16 members per block), each of which fits one of the patterns in Figure 6.

Proof. We begin the proof by considering any triple $\tau_{n}$ such that $\tau_{n}$ is in class 2 , $n^{*}$ is odd, $u\left(n^{*}-2\right)>u\left(n^{*}\right)$, the index of $\gamma_{n 0}$ is equal to -1 and $\theta^{-1}<-E\left(n^{*}\right)<$ $2 \theta^{8}\left|\theta^{\prime}\right|^{3}$. (This is the restriction given in Table 6 for pattern A.) For example, $\tau_{17}$ is such a triple with $n^{*}=-7, u\left(n^{*}\right)=2<u\left(n^{*}-2\right)=3, \gamma_{17,0}=\theta|\varphi|^{-7}$ (see Tables 1 and 2) and $E\left(n^{*}\right)=E(-7)=\theta^{-12} \theta^{\prime-3} \approx-.8026$. 
TABlE 6. Patterns of triples $\tau_{n+j}(0 \leqslant j \leqslant 10$ or $0 \leqslant j \leqslant 15)$

\begin{tabular}{|c|c|c|}
\hline Pattern & Restrictions on $E\left(n^{*}\right)$ & $\begin{array}{c}\text { Associated triples } T_{n+j} \\
\text { and subclass of } T_{n+j}\end{array}$ \\
\hline A & $\begin{aligned} \theta^{-1} & \approx .8019<-\mathrm{E}\left(\mathrm{n}^{*}\right)<1.0306 \approx 2 \theta^{8}\left|\theta^{\prime}\right|^{3} \\
\theta^{-3}\left|\theta^{\prime}\right|^{-1} & \approx 1.1588<\mathrm{E}\left(\mathrm{n}^{*}-1\right)<1.4893 \approx 2 \theta^{6} \theta^{\prime 2} \\
\theta^{-4} & \approx .4136<-\mathrm{E}\left(\mathrm{n}^{*}-2\right)<.5315 \approx 2 \theta^{5}\left|\theta^{\prime}\right|^{3} \\
\theta^{-6}\left|\theta^{\prime}\right|^{-1} & \approx .5976<\mathrm{E}\left(\mathrm{n}^{*}-3\right)<.7681 \approx 2 \theta^{3} \theta^{\prime}{ }^{2} \\
\theta^{-8} \theta^{\prime}-2 & \approx .8636<-\mathrm{E}\left(\mathrm{n}^{*}-4\right)<1.1099 \approx 2 \theta\left|\theta^{\prime}\right| \\
\theta^{-10}\left|\theta^{\prime}\right|^{-3} & \approx 1.2480<\mathrm{E}\left(\mathrm{n}^{*}-5\right)<1.6039 \approx \mathrm{E}_{+}=2 \theta^{-1}\end{aligned}$ & $\begin{array}{l}\tau_{n}, 2 a \\
\tau_{n+3}, 1 a \\
\tau_{n+1}, 3 a ; \tau_{n+2}, 6 a \\
\tau_{n+4}, 5 ; \tau_{n+5}, 8 ; \tau_{n+8}, 1 b \\
\tau_{n+6}, 3 b ; \tau_{n+7}, 6 b \\
\tau_{n+9}, 7 b ; \tau_{n+10}, 4 b\end{array}$ \\
\hline B & $\begin{array}{l}2 \theta^{8}\left|\theta^{\prime}\right|^{3} \approx 1.0306<-\mathrm{E}\left(\mathrm{n}^{*}\right)<1.1099 \approx 2 \theta\left|\theta^{\prime}\right| \\
2 \theta^{6} \theta^{\prime 2} \approx 1.4893<\mathrm{E}\left(\mathrm{n}^{*}-1\right)<1.6039 \approx \mathrm{E}_{+}=2 \theta^{-1} \\
2 \theta^{5}\left|\theta^{\prime}\right|^{3} \approx .5315<-\mathrm{E}\left(\mathrm{n}^{*}-2\right)<.5724 \approx 2 \theta^{-2}\left|\theta^{\prime}\right| \\
2 \theta^{3} \theta^{\prime 2} \approx .7681<\mathrm{E}\left(\mathrm{n}^{*}-3\right)<.8272 \approx 2 \theta^{-4} \\
2 \theta\left|\theta^{\prime}\right| \approx 1.1099<-\mathrm{E}\left(\mathrm{n}^{*}-4\right)<1.1953 \approx 2 \theta^{-6}\left|\theta^{\prime}\right|^{-1} \\
2 \theta^{\prime 2}=\mathrm{E}_{-} \approx .3961<\mathrm{E}\left(\mathrm{n}^{*}-5\right)<.4266 \approx 2 \theta^{-7}\end{array}$ & $\begin{array}{l}\tau_{n}, 2 a \\
\tau_{n+3}, 1 a \\
\tau_{n+1}, 3 a ; \tau_{n+2}, 6 a \\
\tau_{n+4}, 5 ; \tau_{n+5}, 8 ; \tau_{n+8}, 1 b \\
\tau_{n+6}, 3 b ; \tau_{n+7}, 6 b \\
\tau_{n+9}, 7 a ; \tau_{n+10}, 4 a\end{array}$ \\
\hline $\mathrm{Cl}$ & $\begin{array}{c}2 \theta\left|\theta^{\prime}\right| \approx 1.1099<-\mathrm{E}\left(\mathrm{n}^{*}\right)<1.4439 \approx \theta^{9} \theta^{\prime 2} \\
2 \theta^{\prime 2}=\mathrm{E}-.3961<\mathrm{E}\left(\mathrm{n}^{*}-1\right)<.5153 \approx \theta^{8}\left|\theta^{\prime}\right|^{3} \\
2 \theta^{-2}\left|\theta^{\prime}\right| \approx .5724<-\mathrm{E}\left(\mathrm{n}^{*}-2\right)<.7447 \approx \theta^{6} \theta^{\prime}{ }^{2} \\
2 \theta^{-4} \approx .8272<\mathrm{E}\left(\mathrm{n}^{*}-3\right)<1.0761 \approx \theta^{4}\left|\theta^{\prime}\right| \\
2 \theta^{-6}\left|\theta^{\prime}\right|^{-1} \approx 1.1953<-\mathrm{E}\left(\mathrm{n}^{*}-4\right)<1.5550 \approx \theta^{2} \\
2 \theta^{-7} \approx .4266<\mathrm{E}\left(\mathrm{n}^{*}-5\right)<.5550 \approx \theta\left|\theta^{\prime}\right|\end{array}$ & $\begin{array}{l}\tau_{n}, 2 a \\
\tau_{n+3}, 1 c \\
\tau_{n+1}, 3 a ; \tau_{n+2}, 6 a \\
\tau_{n+4}, 5 ; T_{n+5}, 8 ; T_{n+8}, 1 b \\
\tau_{n+6}, 3 b ; T_{n+7}, 6 b \\
\tau_{n+9}, 7 a ; \tau_{n+10}, 4 a\end{array}$ \\
\hline C2 & $\begin{array}{c}\theta^{9} \theta^{\prime 2} \approx 1.4439<-E\left(n^{*}\right)<1.4893 \approx 2 \theta^{6} \theta^{\prime 2} \\
\theta^{8}\left|\theta^{\prime}\right|^{3} \approx .5153<E\left(n^{*}-1\right)<.5315 \approx 2 \theta^{5}\left|\theta^{\prime}\right|^{3} \\
\theta^{6} \theta^{\prime 2} \approx .7447<-E\left(n^{*}-2\right)<.7681 \approx 2 \theta^{3} \theta^{\prime 2} \\
\theta^{4}\left|\theta^{\prime}\right| \approx 1.0761<E\left(n^{*}-3\right)<1.1099 \approx 2 \theta\left|\theta^{\prime}\right| \\
\theta^{2} \approx 1.5550<-E\left(n^{*}-4\right)<1.6039 \approx E_{+}=2 \theta^{-1} \\
\theta\left|\theta^{\prime}\right| \approx .5550<E\left(n^{*}-5\right)<.5724 \approx 2 \theta^{-2}\left|\theta^{\prime}\right| \\
\theta^{-1} \approx .8019<-E\left(n^{*}-6\right)<.8272 \approx 2 \theta^{-4} \\
\theta^{-3}\left|\theta^{\prime}\right|^{-1} \approx 1.1588<E\left(n^{*}-7\right)<1.1953 \approx 2 \theta^{-6}\left|\theta^{\prime}\right|^{-1}\end{array}$ & $\begin{array}{l}\tau_{n}, 2 a \\
\tau_{n+3}, 1 c \\
\tau_{n+1}, 3 a ; \tau_{n+2}, 6 a \\
\tau_{n+4}, 5 ; \tau_{n+5}, 8 ; \tau_{n+8}, 1 a \\
\tau_{n+6}, 3 b ; \tau_{n+7}, 6 b \\
\tau_{n+9}, 5 ; \tau_{n+10}, 8 ; \tau_{n+13}, 1 b \\
\tau_{n+11}, 3 b ; \tau_{n+12}, 6 b \\
\tau_{n+14}, 7 b ; \tau_{n+15}, 4 b\end{array}$ \\
\hline D & $\begin{array}{c}2 \theta^{6} \theta^{\prime 2} \approx 1.4893<-\mathrm{E}\left(\mathrm{n}^{*}\right)<1.5550 \approx \theta^{2} \\
2 \theta^{5}\left|\theta^{\prime}\right|^{3} \approx .5315<\mathrm{E}\left(\mathrm{n}^{*}-1\right)<.5550 \approx \theta\left|\theta^{\prime}\right| \\
2 \theta^{3} \theta^{\prime}{ }^{2} \approx .7681<-\mathrm{E}\left(\mathrm{n}^{*}-2\right)<.8019 \approx \theta^{-1} \\
2 \theta\left|\theta^{\prime}\right| \approx 1.1099<\mathrm{E}\left(\mathrm{n}^{*}-3\right)<1.1588 \approx \theta^{-3}\left|\theta^{\prime}\right|^{-1} \\
2 \theta^{\prime 2}=\mathrm{E} \approx .3961<-\mathrm{E}\left(\mathrm{n}^{*}-4\right)<.4136 \approx \theta^{-4} \\
2 \theta^{-2}\left|\theta^{\prime}\right| \approx .5724<\mathrm{E}\left(\mathrm{n}^{*}-5\right)<.5976 \approx \theta^{-6}\left|\theta^{\prime}\right|^{-1} \\
2 \theta^{-4} \approx .8272<-\mathrm{E}\left(\mathrm{n}^{*}-6\right)<.8636 \approx \theta^{-8} \theta^{\prime}-2 \\
2 \theta^{-6}\left|\theta^{\prime}\right|^{-1} \approx 1.1953<\mathrm{E}\left(\mathrm{n}^{*}-7\right)<1.2480 \approx \theta^{-10}\left|\theta^{\prime}\right|^{-3}\end{array}$ & $\begin{array}{l}\tau_{n}, 2 a \\
\tau_{n+3}, 1 c \\
\tau_{n+1}, 3 a ; \tau_{n+2}, 6 a \\
\tau_{n+4}, 5 ; \tau_{n+5}, 8 ; \tau_{n+8}, 1 a \\
\tau_{n+6}, 3 a ; \tau_{n+7}, 6 a \\
\tau_{n+9}, 5 ; \tau_{n+10}, 8 ; \tau_{n+13}, 1 b \\
\tau_{n+11}, 3 b ; \tau_{n+12}, 6 b \\
\tau_{n+14}, 7 b ; \tau_{n+15}, 4 b\end{array}$ \\
\hline
\end{tabular}

It follows from Lemma 5 that $u\left(n^{*}\right)=u\left(n^{*}-1\right)$ and $u\left(n^{*}-j\right)=u\left(n^{*}-1\right)+1$ for $2 \leqslant j \leqslant 5$; that is, $u\left(n^{*}\right)$ begins a pattern $\mathrm{A}$, as defined above. This means $E\left(n^{*}-2\right)=\theta^{-3} E\left(n^{*}\right) \approx .5157 E\left(n^{*}\right)$, so $\tau_{n+1}$ meets all the requirements of Table 5 for class $3 \mathrm{a}$; note that the fact that

$$
\gamma_{n+1,0}=\theta^{2} \varphi^{-2} \gamma_{n, 0}=\theta^{u(n *-2)}|\varphi|^{n *-2}
$$

which shows that the parity of $(n+1)^{*}$ is odd and that the index of $\gamma_{n+1,0}$ is 0 , follows from the last column of Table 4 , and the relevant entry in that column is determined solely by the fact that $\tau_{n}$ is in class 2 . 
Using Lemma 5 and the last column of Table 4 as we did above, we deduce that $\tau_{n+2}, \tau_{n+3}, \ldots, \tau_{n+10}$ are in classes $6 \mathrm{a}, 1 \mathrm{a}, 5,8,3 \mathrm{~b}, 6 \mathrm{~b}, 1 \mathrm{~b}, 7 \mathrm{~b}, 4 \mathrm{~b}$, respectively. We further deduce that the classes of $\tau_{n+j}, 0 \leqslant j \leqslant 10$, are arranged as in pattern $\mathrm{A}$ of Figure 6 , and that the corresponding values of $i^{*}$ and $E\left(i^{*}\right)(i=n+j$ for some $j)$ satisfy all the relevant conditions in Table 5.

Next we consider any triple $\tau_{n}$ such that $\tau_{n}$ is in class $2, n^{*}$ is odd, $u\left(n^{*}-2\right)>$ $u\left(n^{*}\right)$, the index of $\gamma_{n 0}$ is equal to -1 and $2 \theta^{8}\left|\theta^{\prime}\right|^{3}<-E\left(n^{*}\right)<2 \theta\left|\theta^{\prime}\right|$. (This is the restriction given in Table 6 for pattern B.) Reasoning as above, we deduce that $u\left(n^{*}\right)$ begins a pattern B and that $\tau_{n}, \tau_{n+1}, \ldots, \tau_{n+10}$ are in classes 2a, 3a, 6a, 1a, 5, 8, $3 \mathrm{~b}, 6 \mathrm{~b}, 1 \mathrm{~b}, 7 \mathrm{a}, 4 \mathrm{a}$, respectively. Also as above, we find that the classes of $\tau_{n+j}, 0 \leqslant$ $j \leqslant 10$, are arranged as in pattern B of Figure 6 , and that the corresponding values of $i^{*}$ and $E\left(i^{*}\right)(i=n+j$ for some $j)$ satisfy all the relevant conditions in Table 5 .

The three remaining patterns $\mathrm{C} 1, \mathrm{C} 2, \mathrm{D}$ in Figure 6 are handled the same way: We consider any triple $\tau_{n}$ such that $\tau_{n}$ is in class $2, n^{*}$ is odd, $u\left(n^{*}-2\right)>u\left(n^{*}\right)$, the index of $\gamma_{n 0}$ is -1 and $E\left(n^{*}\right)$ satisfies the restriction given in Table 6 for pattern $\mathrm{C} 1$, $\mathrm{C} 2$ or D. We deduce that $u\left(n^{*}\right)$ begins a pattern $\mathrm{C} 1, \mathrm{C} 2$ or $\mathrm{D}$ and that $\tau_{n+j}(0 \leqslant j$ $\leqslant 10$ for pattern $\mathrm{C} 1,0 \leqslant j \leqslant 15$ for patterns $\mathrm{C} 2$ and $\mathrm{D}$ ) are in the appropriate classes as listed in Table 6. We also find that the classes of $\tau_{n+j}$ are arranged as in the relevant pattern $\mathrm{C} 1, \mathrm{C} 2$ or $\mathrm{D}$ of Figure 6 , and that the corresponding values of $i^{*}$ and $E\left(i^{*}\right)(i=n+j$ for some $j)$ satisfy all the relevant conditions in Table 5 .

We have now shown that whenever $\tau_{n}$ is in class $2, n^{*}$ is odd, $u\left(n^{*}-2\right)>u\left(n^{*}\right)$, the index of $\gamma_{n 0}$ is equal to -1 and $\theta^{-1}<-E\left(n^{*}\right)<\theta^{2}$, then $\tau_{n}$ begins one of the patterns $\mathrm{A}, \mathrm{B}, \mathrm{C} 1, \mathrm{C} 2$ or $\mathrm{D}$ of Table 6 . However, we see from Table 6 that all of these patterns end with a triple $\left(\tau_{n+10}\right.$ for patterns A, B, C1 and $\tau_{n+15}$ for patterns C2, D) which is in class 4 . By Theorem 4, the next triple, say $\tau_{j}$ (so $j=n+11$ for patterns $\mathrm{A}, \mathrm{B}, \mathrm{C} 1$ and $j=n+16$ for patterns $\mathrm{C} 2, \mathrm{D})$, is in class 2 , and we see from Table 6 that $j^{*}$ is odd, $u\left(j^{*}-2\right)>u\left(j^{*}\right)$ (for this we also need Lemmas 4 and 5), the index of $\gamma_{j 0}$ is equal to -1 and $\theta^{-1}<-E\left(j^{*}\right)<\theta^{2}$. It follows that each of the patterns $\mathbf{A}, \mathbf{B}$, $\mathrm{C} 1, \mathrm{C} 2$, D leads into another one of these five patterns; the pattern which follows a given pattern for $\tau_{n}, \tau_{n+1}, \ldots, \tau_{n+j}(j=10$ or 15$)$ is determined by the value of $E\left(n^{*}\right)$, as indicated in Table 7 .

Hence we have proved that as soon as the triples $\tau_{n}$ fall into one of the five patterns, the rest of the sequence of triples can be divided into a sequence of blocks, each of which fits one of the patterns. We have already seen that the first pattern (which turns out to be pattern A) begins with $\tau_{17}$.

We have now proved all of the assertions in Theorem 5, except for the deduction of the restrictions on $E\left(n^{*}\right)$ in Table 5 from the restrictions on $E\left(n^{*}\right)$ given in Table 6. This is, however, easily explained as follows: We observe that class $3 a$, for example, occurs in position $n^{*}-2$ in patterns A, B, C1, C2 of Figure 6, and in both of the positions $n^{*}-2$ and $n^{*}-4$ in pattern $\mathrm{D}$ of Figure 6 . If we look at the corresponding six inequalities for $E\left(n^{*}-j\right)$ (where $j$ is 2 or 4 , as appropriate) in Table 6 , we find that together these inequalities cover the interval $E_{-}<-E\left(n^{*}\right)<\theta^{-1}$. This is exactly the interval for class 3a given in Table 5. We prove all of the other restrictions on $E\left(n^{*}\right)$ 


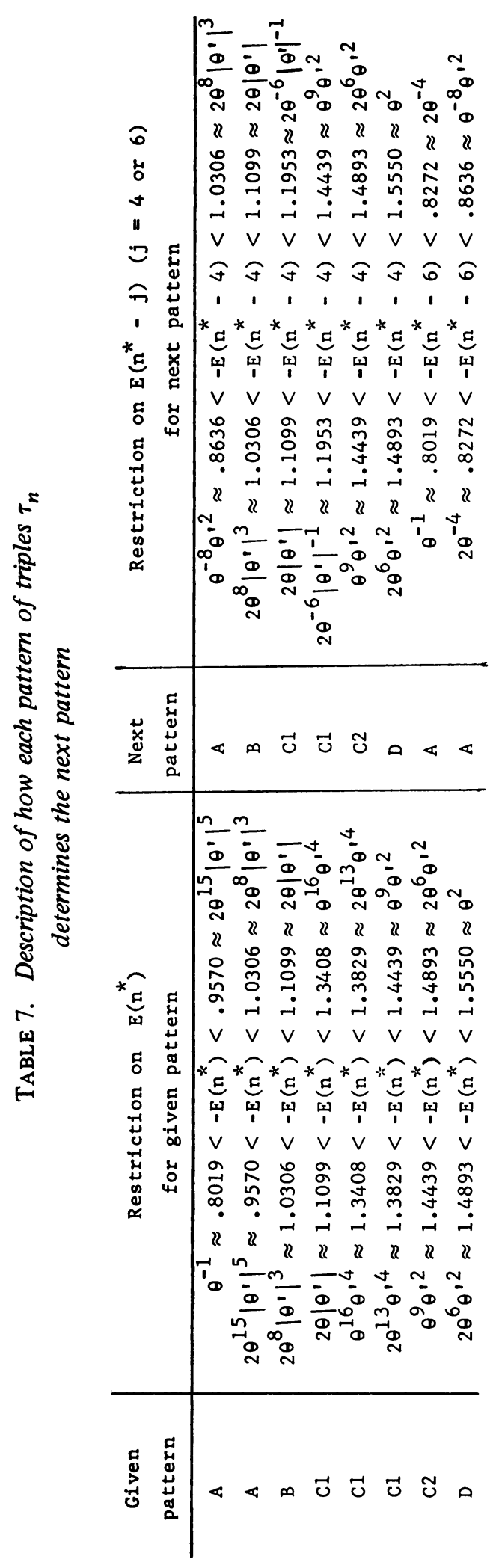


of Table 5 in an analogous manner (except for classes 9 and 10, which do not occur in any of the patterns of Figure 6; for these we can give an ad hoc proof very similar to the proofs for the other classes). This completes the proof of Theorem 5 .

We are now in a position to prove the following result, which, as mentioned in Section 1, verifies a conjecture of Szekeres.

THEOREM 6. The linear forms $\gamma_{n 0}(n=0,1,2, \ldots)$ given by the 2-fraction for $(\alpha, \beta)$ include all of the best approximations to zero by the linear form $x_{0}+x_{1} \alpha$ $+x_{2} \beta$.

Proof. Theorem 2 gives necessary conditions for a best approximation. If $\gamma_{n 0}$ is a best approximation, then by (4.3) one of the five conditions of Theorem 2 must hold with $n$ replaced by $n^{*}$ and $m$ replaced by the index $i$ of $\gamma_{n 0}$. Thus, in order to prove Theorem 6 it suffices to show that for each of the five conditions of Theorem 2, if there is a best approximation satisfying the condition, then there is always some $\gamma_{j 0}$ for which the condition holds with $n$ replaced by $j^{*}$ and $m$ replaced by the index $i$ of $\gamma_{j 0}$.

If $j^{*}$ is even and $i=-1$ or 0 , then we see from Figure 6 that the 2 -fraction algorithm always provides a suitable $\gamma_{j 0}$. This covers condition (i) of Theorem 2. If $j^{*}$ is even and $i=1$, then we see from Figure 6 that the 2 -fraction does not provide a suitable $\gamma_{j 0}$ only in the following cases: pattern $\mathrm{B}, j^{*}=n^{*}-5$; pattern $\mathrm{C} 1, j^{*}=n^{*}-$ 1 or $n^{*}-5$; pattern $\mathrm{C} 2, j^{*}=n^{*}-1$; and pattern $\mathrm{D}, j^{*}=n^{*}-1$. However, according to Table 6 , in none of these cases is the necessary condition $E\left(j^{*}\right)>\left|\theta^{3} \theta^{\prime}\right|^{-1} \approx 1.1588$ of Theorem 2, (ii) satisfied. Thus the 2-fraction covers condition (ii) of Theorem 2.

If $j^{*}$ is odd and $i=1$, then it follows from Figure 6 that the 2 -fraction does not provide a suitable $\gamma_{j 0}$ only in the following cases: patterns $\mathrm{A}, \mathrm{B}, \mathrm{C} 1$ and $\mathrm{C} 2, j^{*}=n^{*}-$ 2; and pattern $\mathrm{D}, j^{*}=n^{*}-2$ or $n^{*}-4$. But by Table 6 , in none of these cases is the necessary condition $-E\left(j^{*}\right)>\theta^{-1} \approx .8019$ of Theorem 2 , (iv) satisfied. Thus the 2 fraction covers condition (iv) of Theorem 2 .

If $j^{*}$ is odd and $i=2$, then we see from Figure 6 that the 2-fraction never provides a suitable $\gamma_{j 0}$. However, in only one case does it happen that the necessary condition $-E\left(j^{*}\right)>\theta^{2} \approx 1.5550$ of Theorem 2 , (v) is satisfied when $j^{*}$ is odd: namely, as we see from Table 6 , for pattern $\mathrm{C} 2, j^{*}=n^{*}-4$. It turns out that in this case $\left|R\left(u\left(j^{*}\right)+2, j^{*}\right)\right|$ is never a best approximation (that is, the necessary condition of Theorem 2, (v) is not sufficient in this case). We see this as follows: We have

$$
\left|R\left(u\left(j^{*}\right)+2, j^{*}\right)\right|=b\left(u\left(j^{*}\right)+2, j^{*}\right)+k\left(u\left(j^{*}\right)+2, j^{*}\right) \alpha+g\left(u\left(j^{*}\right)+2, j^{*}\right) \beta
$$

by (3.5). We also have $T_{2, j^{*}}=\left|k\left(u\left(j^{*}\right)+2, j^{*}\right)\right|$ by [3, second line of formula (12), p. 983] (recall from Section 3 that we must replace the notation $T_{m, n}$ of [3] by $\left.T_{m+1, n}\right)$. We shall prove that $\left|R\left(u\left(j^{*}\right)+2, j^{*}-1\right)\right|$ satisfies $u\left(j^{*}-1\right)=u\left(j^{*}\right)+1$ and $T_{1, j^{*}-1}=T_{2, j^{*}}$. Since

$$
\left|R\left(u\left(j^{*}\right)+2, j^{*}-1\right)\right|=|\varphi|^{-1}\left|R\left(u\left(j^{*}\right)+2, j^{*}\right)\right|<\left|R\left(u\left(j^{*}\right)+2, j^{*}\right)\right|
$$

by (4.3), it will follow that $\left|R\left(u\left(j^{*}\right)+2, j^{*}\right)\right|$ cannot be a best approximation (using the definition of best approximation given in Section 2). The assertion $u\left(j^{*}-1\right)=$ $u\left(j^{*}\right)+1$ follows from Figure 6 , since $j^{*}=n^{*}-4$ and we are in pattern C2. The 
assertion $T_{1, j^{*}-1}=T_{2, j^{*}}$ follows from the facts $T_{1, j^{*}-1}=\left|g\left(u\left(j^{*}-1\right)+1, j^{*}-1\right)\right|$ [3, third line of formula (12), p. 983] and

$$
\left|g\left(u\left(j^{*}-1\right)+1, j^{*}-1\right)\right|=\left|k\left(u\left(j^{*}\right)+2, j^{*}\right)\right| .
$$

(This is a special case of the general fact that $|g(m, n-1)|=|k(m, n)|$; see the explanation in [3, p. 980].) This completes the proof that no best approximation satisfying condition (v) of Theorem 2 is not of the form $\gamma_{j 0}$ (of course, what we actually showed is that there are no best approximations satisfying condition (v) of Theorem 2!).

If $j^{*}$ is odd and $i=0$, then it follows from Figure 6 that the 2 -fraction always provides a suitable $\gamma_{j 0}$. If $j^{*}$ is odd and $i=-1$, then it follows from Figure 6 that the 2 -fraction does not provide a suitable $\gamma_{j 0}$ only in the case of pattern $\mathrm{C} 2, j^{*}=n^{*}-4$. We show that in this case $\left|R\left(u\left(j^{*}\right)-1, j^{*}\right)\right|$ cannot provide a best approximation, because

$$
\left|R\left(u\left(j^{*}\right)+2, j^{*}-1\right)\right|=\theta^{-3}|\varphi|^{-1}\left|R\left(u\left(j^{*}\right)-1, j^{*}\right)\right|<\left|R\left(u\left(j^{*}\right)-1, j^{*}\right)\right|,
$$

$u\left(j^{*}-1\right)=u\left(j^{*}\right)+1\left(\right.$ from Figure 6 , since $j^{*}=n^{*}-4$ and we are in pattern $\left.\mathrm{C} 2\right)$ and

$$
T_{1, j^{*}-1}=T_{2, j^{*}}<T_{0, j^{*}}<T_{-1, j^{*}}
$$

(the equality here was proved in the preceding paragraph; the first inequality always holds when $\left|E\left(j^{*}\right)\right|>\theta^{2}$ by [3, Lemma 9 and Corollary 2, p. 990], and $\left|E\left(j^{*}\right)\right|>\theta^{2}$ holds for pattern $\mathrm{C} 2, j^{*}=n^{*}-4$, by Table 6 ; the second inequality holds by (3.9) of Lemma 3). Thus we have proved that any best approximation satisfying condition (iii) of Theorem 2 must be given by some $\gamma_{j 0}$. This completes the proof of Theorem 6 , except for the fact that we have so far tacitly assumed that $\left|j^{*}\right|$ is large (because $|n|$ is assumed large in Theorem 2, for example). However, calculation of the first few best approximations to zero by $x_{0}+x_{1} \alpha+x_{2} \beta$ shows that all of them are of the form $\gamma_{n 0}$. This means Theorem 6 holds as stated.

5. Almost Periodicity. It follows from Figure 6 and the last sentence of Theorem 5 that for $n \geqslant 17$ we never have $\epsilon(n)=\epsilon(n+1)=0$. Table 2 shows that in fact for $n \geqslant 0, \epsilon(n)$ never has the value 0 for two consecutive values of $n$. By the definitions (2.2) and (2.3), this means that the digits $d_{i}$ of the 2-fraction for $(\alpha, \beta)$ are all either 1 or 2. Furthermore, we find that after some initial irregularities (a close look at Table 2 shows that the irregularities end at $d_{13}=1$ ), the sequence $d_{1}, d_{2}, \ldots$ is made up of repetitions of the digit blocks

$$
a=2121 \text { and } b=1121 .
$$

Thus we have proved some of the characteristics of the sequence $d_{1}, d_{2}, \ldots$ which were first mentioned at the end of Section 2.

Using the work of Section 4, we will also be able to prove that the sequence of digits $d_{i}$ is almost periodic, in a suitable sense. The rest of this section is devoted to exploring this almost periodicity.

The definition of almost periodicity that we shall use is the following: We say the sequence of integers $n_{1}, n_{2}, \ldots$ is almost periodic if there exists a subscript $I$ such that given any $N \geqslant 1$, there exists $M>N$ for which $n_{I+i}=n_{I+M+i}, 1 \leqslant i \leqslant N$, holds. 
THEOREM 7. The sequence $d_{1}, d_{2}, \ldots$ of digits of the 2-fraction for $(\alpha, \beta)$ is almost periodic.

Proof. Using Table 2, we see that the sequence $d_{1}, d_{2}, \ldots$ begins

$$
2,1,1,1,1,2,1,2,1,2,1,1,1, a, b, a, b .
$$

(Here we use the abbreviations (5.1).) By Theorem 5, the sequence $d_{i}, i \geqslant 14$, is a sequence of digit blocks $a$ and $b$.

Using Theorem 5 and Table 7 , we can easily prove that $d_{1}, d_{2}, \ldots$ satisfies the definition of an almost periodic sequence with $I=13$ (to dispose of the initial irregularities shown in (5.2)). For it follows from Theorem 5 and Table 7 that if we have a given pattern $\mathrm{A}, \mathrm{B}, \mathrm{C} 1, \mathrm{C} 2$ or $\mathrm{D}$ made up of $\tau_{n}, \tau_{n+1}, \ldots, \tau_{n+j}(j=10$ or 15$)$, then the value of $E\left(n^{*}\right)$ by itself determines what the next pattern (say it is made up of $\tau_{m}, \tau_{m+1}, \ldots$, etc.) will be, and also determines (by repeated use of Lemma 5; recall the proof of the inequalities in Table 6) the value of $E\left(m^{*}\right)$. Hence, if $E\left(n^{*}\right)$ has a given value at the beginning of the first member of a sequence of patterns (for example, the sequence $\mathrm{A}, \mathrm{A}, \mathrm{A}, \mathrm{A}, \mathrm{B}, \mathrm{C} 1, \mathrm{C} 1, \mathrm{C} 1, \mathrm{C} 2$ ), then there is an $\epsilon>0$ such that whenever $m^{*}$ satisfies $\left|E\left(m^{*}\right)-E\left(n^{*}\right)\right|<\epsilon$ and $\tau_{m}$ is in class 2 , then $\tau_{m}$ is the first triple in the first member of an identical sequence of patterns. It follows easily from Kronecker's theorem on inhomogeneous Diophantine approximation (see Koksma [5, p. 83]) that $-E(n), n$ odd $(n=-1,-3,-5, \ldots)$ is dense in the interval $\left[E_{-}, E_{+}\right]$. Hence given any value of $E\left(n^{*}\right), n^{*}$ odd, we can find a value of $E\left(m^{*}\right), m$ odd, which is as close to $E\left(n^{*}\right)$ as we wish. Now the almost periodicity of the sequence of $d_{i}$ 's follows at once from our previous remarks.

Of course, Theorem 7 does not imply that the sequence of $d_{i}$ 's follows the very special almost periodic pattern obtained by extending (2.23) via the partial quotients of the number $\eta$ defined in (2.25) (see the end of Section 2 for an explanation of this special pattern). Indeed, the question of whether this special pattern is actually followed by the $d_{i}$ 's is too delicate to be decided here. All that we do here is to prove a certain consequence of assuming that the special pattern is followed. To state this, we need to define

$$
N_{a}(n)=\text { number of digit blocks } a \text { contained in } d_{14}, d_{15}, \ldots, d_{n}
$$

and

$$
N_{b}(n)=\text { number of digit blocks } b \text { contained in } d_{14}, d_{15}, \ldots, d_{n} .
$$

The fact we shall prove is the conclusion of the next lemma.

Lемма 7. Suppose that, after the initial irregularities, for any $n$ the sequence $d_{1}, d_{2}, \ldots, d_{n}$ is contained in some $s_{j}$, where the first few values of $s_{i}$ are defined by (2.23) and in general $s_{i+1}=s_{i}^{\beta(i)} s_{i-1}$, where the $\beta(i)(i=1,2, \ldots)$ are the partial quotients in the continued fraction for the number $\eta$ defined by (2.25). Then $\eta$ is a limit point of the set $\left\{N_{a}(n) / N_{b}(n): n=21,22, \ldots\right\}$.

Proof. The lemma follows easily from the definition of the $s_{i}$ (see Szekeres [9, p. 139]). Szekeres [9, pp. 138-139] gives a geometric argument for the plausibility of the hypothesis of the lemma. 
Our next lemma shows that we can prove the conclusion of Lemma 7 without deciding whether the hypothesis of Lemma 7 is true.

LEMMA 8. The number $\eta$ defined by (2.25) is a limit point of the set $\left\{N_{a}(n) / N_{b}(n): n=21,22, \ldots\right\}$.

Proof. We know from Theorem 5 and Figure 6 that the sequence $d_{14}, d_{15}, \ldots$ is made up of patterns $\mathrm{A}, \mathrm{B}, \mathrm{Cl}$ (each consisting of a digit block $\boldsymbol{a}$ followed by a digit block $b$ ) and $\mathrm{C} 2$, D (each consisting of a digit block $a$ followed by two digit blocks $b$ ). Let $N(\mathrm{~A}, \mathrm{~B}, \mathrm{C} 1 ; n)$ and $N(\mathrm{C} 2, \mathrm{D} ; n)$ denote the number of patterns $\mathrm{A}, \mathrm{B}, \mathrm{C} 1$ and $\mathrm{C} 2, \mathrm{D}$, respectively, contained in $d_{14}, d_{15}, \ldots, n$; then

$$
\frac{N_{a}(n)}{N_{b}(n)}=\frac{N(\mathrm{~A}, \mathrm{~B}, \mathrm{C} 1 ; n)+N(\mathrm{C} 2, \mathrm{D} ; n)}{N(\mathrm{~A}, \mathrm{~B}, \mathrm{C} 1 ; n)+2 N(\mathrm{C} 2, \mathrm{D} ; n)} .
$$

It follows from (5.3) that the lemma is proved if we can show that $\zeta$ is a limit point of $\{N(\mathrm{~A}, \mathrm{~B}, \mathrm{C} 1 ; n) / N(\mathrm{C} 2, \mathrm{D} ; n)\}$, where $\zeta$ is defined by

$$
\eta=\frac{1}{1+(1+\zeta)^{-1}}
$$

We see from Figure 6 that if $\tau_{n}$ is the first triple of one of the patterns A, B or $\mathrm{C} 1$, with associated value $E\left(n^{*}\right)$, then $\tau_{n+11}$ is the first triple of the next pattern, with associated value $E\left(n^{*}-4\right)$. Similarly, if $\tau_{n}$ is the first triple of one of the patterns C2 or $\mathrm{D}$, then $\tau_{n+16}$ is the first triple of the next pattern, with associated value $E\left(n^{*}-6\right)$. Thus by Table 6 when we pass from a pattern A, B or C1 to the next pattern, the number $E\left(n^{*}\right)$ is multiplied by $\left(\theta^{7} \theta^{\prime 2}\right)^{-1}=Q_{1}^{-1}$, say; and when we pass from a pattern $\mathrm{C} 2$ or $\mathrm{D}$ to the next pattern, the number $E\left(n^{*}\right)$ is multiplied by $\left(\theta^{10} \theta^{\prime 2}\right)^{-1}=Q_{2}^{-1}$, say.

We saw in the proof of Theorem 7 that if $E\left(n^{*}\right)$ is the value associated with the first triple $\tau_{n}$ in the first member of a sequence of patterns (each of which is A, B, C1, $\mathrm{C} 2$ or $\mathrm{D})$, then the sequence of patterns appears again as soon as we reach a triple $\tau_{m}$ such that $\tau_{m}$ is the first triple in a pattern and $E\left(m^{*}\right)$ is sufficiently close to $E\left(n^{*}\right)$. Now let $p=p(n)=N(\mathrm{~A}, \mathrm{~B}, \mathrm{C} 1 ; n)$ and $q=q(n)=N(\mathrm{C} 2, \mathrm{D} ; n)$. It follows from the almost periodicity established in Theorem 7 that for infinitely many $n$ we have

$$
Q_{1}^{p} Q_{2}^{q}=\theta^{7 p+10 q} \theta^{\prime 2 p+2 q} \approx 1
$$

Solving (5.5) for $p / q$ gives

$$
\frac{p}{q} \approx \frac{-10 \log \theta-2 \log \left|\theta^{\prime}\right|}{7 \log \theta+2 \log \left|\theta^{\prime}\right|}=\zeta \approx 7.93576549,
$$

where $\zeta$ is defined by (5.4). Thus $\zeta$ is a limit point of $\{N(\mathrm{~A}, \mathrm{~B}, \mathrm{C} 1 ; n) / N(\mathrm{C} 2, \mathrm{D} ; n)\}$, and Lemma 8 is proved.

\footnotetext{
Department of Mathematics

State University of New York at Buffalo

4246 Ridge Lea Road

Amherst, New York 14226
}

1. ALAN BAKER, "A sharpening of the bounds for linear forms in logarithms," Acta Arith., v. 21, 1972, pp. 117-129. MR 46 \#1717. 
2. T. W. CUSICK, "Diophantine approximation of ternary linear forms," Math. Comp., v. 25, 1971, pp. 163-180. MR 45 \#5083.

3. T. W. CUSICK, "Diophantine approximation of ternary linear forms. II," Math. Comp., v. 26, 1972, pp. 977-993. MR 48 \#44.

4. T. W. CUSICK, "Diophantine approximation of linear forms over an algebraic number field," Mathematika, v. 20, 1973, pp. 16-23. MR $49 \# 4942$.

5. J. F. KOKSMA, Diophantische Approximationen, reprint of 1936 Springer edition, Chelsea, New York.

6. S. LANG, Introduction to Diophantine Approximations, Addison-Wesley, Reading, Mass., 1966. MR 35 \#129.

7. W. J. LeVEQUE (Editor), Reviews in Number Theory, Vol. 1, Amer. Math. Soc., Providence, R. I., 1974. MR 50 \#2040.

8. H. MINKOWSKI, "Zur Theorie der Kettenbruche," in Gesammelte Abhandlungen, Vol. I, Teubner, Leipzig, 1911, pp. 278-292.

9. G. SZEKERES, "Multidimensional continued fractions," Ann. Univ. Sci. Budapest. Eötvös Sect. Math., v. 13, 1970, pp. 113-140. MR 47 \#1753. 